\title{
On Memory Traffic and Optimisations for Low-order Finite Element Assembly Algorithms on Multi-core CPUs
}

\author{
JAMES D. TROTTER and XING CAI, Simula Research Laboratory and University of Oslo \\ SIMON W. FUNKE, Simula Research Laboratory
}

\begin{abstract}
Motivated by the wish to understand the achievable performance of finite element assembly on unstructured computational meshes, we dissect the standard cellwise assembly algorithm into four kernels, two of which are dominated by irregular memory traffic. Several optimisation schemes are studied together with associated lower and upper bounds on the estimated memory traffic volume. Apart from properly reordering the mesh entities, the two most significant optimisations include adopting a lookup table in adding element matrices or vectors to their global counterparts, and using a row-wise assembly algorithm for multi-threaded parallelisation. Rigorous benchmarking shows that, due to the various optimisations, the actual volumes of memory traffic are in many cases very close to the estimated lower bounds. These results confirm the effectiveness of the optimisations, while also providing a recipe for developing efficient software for finite element assembly.

CCS Concepts: • Mathematics of computing $\rightarrow$ Mathematical software performance; ・ Theory of computation $\rightarrow$ Shared memory algorithms;
\end{abstract}

Additional Key Words and Phrases: Finite element methods, assembly, multi-core, Intel Xeon, AMD Epyc, Cavium TX2

\section{ACM Reference format:}

James D. Trotter, Xing Cai, and Simon W. Funke. 2022. On Memory Traffic and Optimisations for Low-order Finite Element Assembly Algorithms on Multi-core CPUs. ACM Trans. Math. Softw. 48, 2, Article 19 (May 2022), 31 pages.

https://doi.org/10.1145/3503925

\section{INTRODUCTION}

Finite element methods are among the most important and widely used techniques for numerically solving partial differential equations (PDEs), especially when the geometry of the solution domain is best described by an unstructured computational mesh. While offering flexibility to handle complicated geometries, unstructured meshes bring challenges with respect to achieving performance on modern computing hardware. In particular, unstructured meshes lead to irregular memory access patterns that are difficult for hierarchical, cache-based memories. This is due to poor data prefetching and limited data reuse in the caches. Another challenge arises with multi-threaded

This work was supported by the Research Council of Norway under contract 251186. Also, the research presented in this article has benefited from the Experimental Infrastructure for Exploration of Exascale Computing (eX3), which is financially supported by the Research Council of Norway under contract 270053 (https:/www.ex3.simula.no/).

Authors' addresses: J. D. Trotter and X. Cai, Simula Research Laboratory, P.O. Box 134, Lysaker 1325, Norway, University of Oslo, Department of Informatics, P.O. Box 1080 Blindern, Oslo 0316, Norway; emails: \{james, xingca\}@simula.no; S. W. Funke, Simula Research Laboratory, P.O. Box 134, Lysaker 1325, Norway; email: simon@simula.no.

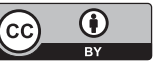

This work is licensed under a Creative Commons Attribution International 4.0 License.

(c) 2022 Copyright held by the owner/author(s).

0098-3500/2022/05-ART19

https://doi.org/10.1145/3503925 
parallel computing because irregular memory accesses can lead to race conditions (i.e., several threads simultaneously update the same variables) that are not easy to determine beforehand.

From a computational point of view, the application of finite element methods to PDEs ultimately translates into assembling systems of linear algebraic equations and then solving them. In many cases, a significant portion of the overall computation occurs during the assembly stage, which is the topic of the current article. Our aim is to understand how memory traffic-the movement of data to and from memory-impacts the performance of such calculations whenever unstructured meshes are used. Generally speaking, it is important to execute the assembly with high parallel performance to prevent it from becoming a bottleneck. For non-linear problems, assembly is also critical to achieving good performance, because it must be carried out during each iteration of a non-linear solver.

Considerable efforts have gone into optimising finite element assembly algorithms [Cantwell et al. 2011; Kronbichler and Kormann 2012; Markall et al. 2013; Vos et al. 2010], particularly in connection with automatically generating high-performance assembly code [Kirby et al. 2005; Kirby and Logg 2006; Luporini et al. 2017, 2015; Ølgaard and Wells 2010; Russell and Kelly 2013; Sun et al. 2020]. However, the emphasis of these efforts is mainly placed on reducing the number of floating point operations performed, without fully accounting for the impact of memory traffic on the performance. For discretisations that are based on low-degree polynomials, such as linear and quadratic finite elements, counting floating point operations alone is not sufficient to understand the achievable performance. Instead, it is important in these cases to have a quantified understanding of the memory traffic and devise optimisation strategies accordingly.

In this article, our initial focus is on the standard cellwise assembly algorithm, which is commonly used for building vectors and sparse matrices from finite element variational forms. We dissect this algorithm into four different kernels and investigate them in detail with respect to the floating point computations and memory traffic involved. The novelty of our work is the derivation of precise lower and upper bounds on memory traffic for the memory-traffic-heavy kernels and several optimisation strategies. These optimisations even include two seemingly memory-wise wasteful schemes, i.e., the use of a lookup table in adding element matrices and vectors to their global counterparts, and a row-wise assembly algorithm. The latter is for avoiding race conditions in the context of a multi-threaded parallel assembly. Our contribution includes a quantification of the effectiveness of the optimisations, by rigorously benchmarking the actual memory traffic volumes (measured by hardware performance counters) against the lower and upper bounds. Our work thus provides a recipe for developing serial and parallel software for low-order finite element assembly algorithms.

The remainder of the article is organised as follows. We begin with some background information on finite element assembly in Section 2. The cellwise assembly algorithm is detailedly dissected in Section 3, whereas a few memory-oriented optimisations, including the row-wise assembly algorithm, are explored in Section 4. In Section 5, we derive precise lower and upper bounds on the memory traffic involved in these calculations. Next, Section 6 presents benchmarking results from various finite element assembly computations. In Section 7, connections are made to relevant work on finite element methods, before we draw our conclusions in Section 8 .

\section{BACKGROUND}

In this section, we briefly present some notation and familiar concepts from the finite element literature that are needed to describe the usual finite element assembly procedure. Further details can be found, for instance, in Ern and Guermond [2004] and Ciarlet [2002]. 
Computations based on finite elements usually begin with a variational formulation, consisting of a bilinear form $a: V \times U \rightarrow \mathbf{R}$ and a linear form $\ell: V \rightarrow \mathbf{R}$, where $V$ and $U$ are called, respectively, the test and trial function spaces. The test and trial space are often one and the same, though, in general, they do not need to be. The objective is to find a solution $u \in U$ such that $a(v, u)=\ell(v)$ for every $v \in V$. If we choose a basis $\left\{\psi_{i}\right\}_{i=0}^{M-1}$ for the test space and another basis $\left\{\phi_{j}\right\}_{j=0}^{N-1}$ for the trial space, then we can compute a linear system of equations, $A x=b$, with the coefficient matrix $A_{i, j}=a\left(\psi_{i}, \phi_{j}\right)$ and right-hand side vector $b_{i}=\ell\left(\psi_{i}\right)$. When the linear system is solved, it yields a solution $u=\sum_{j=0}^{N-1} x_{j} \phi_{j}$ to the variational formulation of the PDE.

The bilinear form $a$ and linear form $\ell$ are primarily defined in terms of integrals over a domain $\Omega \subset \mathbf{R}^{d}, d>0$, though some additional constraints may arise due to handling prescribed boundary conditions. To illustrate, we use a classical example of a linear, elliptic PDE, which is Poisson's equation,

$$
-\nabla \cdot(c(x) \nabla u(x))=f(x) \text { in } \Omega,
$$

with a homogeneous Neumann boundary condition. The function $f \in L^{2}(\Omega)$ represents a given source term, and $c$ is a given, spatially varying coefficient, such that $c(x)>0$. For this problem, the test and trial spaces are finite-dimensional subspaces of the Sobolev space $H^{1}(\Omega)$, and the standard variational form consists of the weak formulation of the Laplacian

$$
a(v, u)=\int_{\Omega} c \nabla v \cdot \nabla u \mathrm{~d} x
$$

and the linear form

$$
\ell(v)=\int_{\Omega} f v \mathrm{~d} x
$$

The basic approach, as outlined above, also applies to systems of PDEs, where the trial and test spaces consist of vector-valued functions, and to time-dependent and non-linear problems, both of which are reduced to solving sequences of linear problems.

In this article, we are interested in the assembly of coefficient matrices that are derived from bilinear variational forms whose test and trial spaces are defined in terms of finite elements. First, a computational mesh, or triangulation, denoted by $\mathcal{T}$, is introduced by partitioning a domain $\Omega$ into cells, usually convex polygons or polyhedra. Next, local function spaces $X(T)$ on each mesh cell $T \in \mathcal{T}$ are combined to form a global finite element space $X(\mathcal{T})$, consisting of functions defined piecewise with respect to the mesh $\mathcal{T}$. Here, we use $\theta_{0}, \theta_{1}, \ldots, \theta_{M-1}$ to denote the basis functions of the global finite element space $X(\mathcal{T})$. For each mesh cell $T$, there is an injective map $\mu:[0, m) \rightarrow$ $[0, M)$, called a local-to-global mapping, that identifies each basis function $\theta_{0}^{T}, \theta_{1}^{T}, \ldots, \theta_{m-1}^{T}$ of the local space $X(T)$ with a corresponding global basis function, such that $\theta_{\mu(j)}(x)=\theta_{j}^{T}(x)$ whenever $x \in T$, for $0 \leq j<m$. For simplicity, we restrict our attention to polynomial spaces and the commonly used Lagrange elements, whose basis consists of Lagrange interpolating polynomials on each mesh cell.

The most common approach is to use a cellwise assembly algorithm that computes the matrix $A$ as a sum over the mesh cells,

$$
A=\sum_{T \in \mathcal{T}} P_{T} A_{T} Q_{T}^{T}
$$

Denoting $m=\operatorname{dim} V(T)$ and $n=\operatorname{dim} U(T)$, then $A_{T} \in \mathbf{R}^{m, n}$ is an element matrix for a mesh cell $T$,

$$
\left(A_{T}\right)_{i, j}=a\left(\tilde{\psi}_{i}, \tilde{\phi}_{j}\right)
$$

where $\tilde{\psi}_{i} \in V(T)$ and $\tilde{\phi}_{j} \in U(T)$ are local basis functions. The purpose of the matrices $P_{T} \in \mathbf{R}^{M, m}$ and $Q_{T} \in \mathbf{R}^{N, n}$ is to "scatter" contributions from the element matrix $A_{T}$ to the correct locations 
of the global matrix $A$. More specifically, if $\mu$ and $v$ denote local-to-global mappings of the test and trial space, respectively, then $\left(P_{T}\right)_{i, j}=\delta_{i, \mu(j)}$ and $\left(Q_{T}\right)_{i, j}=\delta_{i, v(j)}$, where $\delta_{i, j}$ denotes the Kronecker delta function.

\section{CELLWISE FINITE ELEMENT ASSEMBLY}

For a global matrix, $A$, corresponding to some bilinear variational form and a mesh $\mathcal{T}$, the highlevel pseudo-code in Algorithm 1 describes a typical cellwise assembly algorithm. The calculations pertaining to each mesh cell can thus be dissected into four separate parts, or kernels, each of which is described in detail in the four following subsections. The final subsection describes a straightforward parallelisation of the cellwise assembly algorithm.

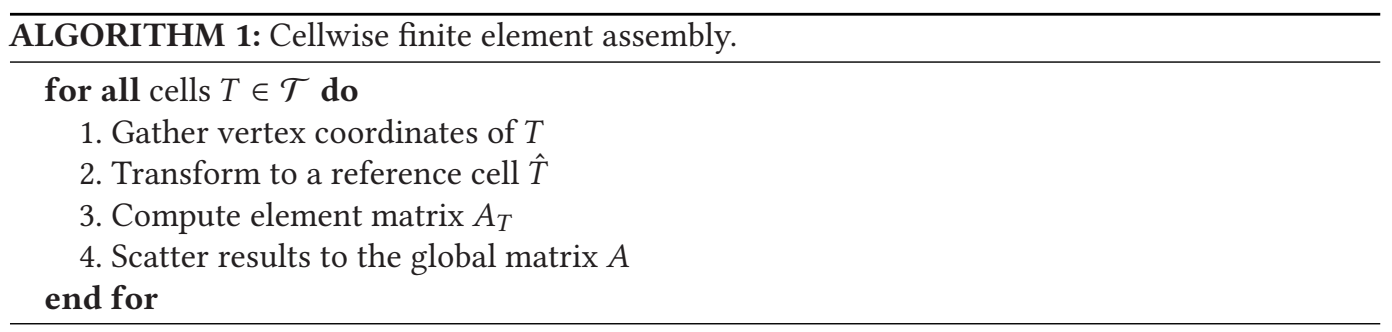

\subsection{Gathering Vertex Coordinates}

The first step is to gather the coordinates of a cell's vertices from a data structure storing the entire mesh $\mathcal{T}$. The vertex coordinates are later used to compute the transformation to a reference cell and the element matrix. In general, an unstructured mesh is used, thus an explicit representation of the connectivity between mesh cells is needed. In this article, we primarily consider three-dimensional meshes whose cells are tetrahedra, though the following description extends easily to other commonly used mesh cells, such as quadrilaterals or hexahedra.

Let $\Omega \subset \mathbf{R}^{d}(d=3)$ denote a bounded, polyhedral domain, and let $\mathcal{T}$ be a mesh with vertices $p_{0}, p_{1}, \ldots, p_{M-1} \in \mathbf{R}^{d}$ that partitions $\Omega$ into the $d$-dimensional mesh cells, $T_{0}, T_{1}, \ldots, T_{N-1}$. Each cell $T$ is defined by $m$ distinct vertices, $p_{\sigma(0)}, p_{\sigma(1)}, \ldots, p_{\sigma(m-1)}$, where $\sigma:[0, m) \rightarrow[0, M)$ is an injective map.

If we consider all the vertex coordinates of the mesh $\mathcal{T}$ as an $M \times d$ matrix,

$$
\left[\begin{array}{cccc}
p_{0,0} & p_{0,1} & \ldots & p_{0, d-1} \\
p_{1,0} & p_{1,1} & \ldots & p_{1, d-1} \\
\vdots & \vdots & \ddots & \vdots \\
p_{M-1,0} & p_{M-1,1} & \ldots & p_{M-1, d-1}
\end{array}\right],
$$

then these values are typically stored in memory in a row-major order. The vertex coordinates are usually not accessed in sequence. Instead, gathering the coordinates of a cell $T=$ $\left[p_{\sigma(0)} \ldots p_{\sigma(m-1)}\right]$ requires loading $m$ different rows, $\sigma(0), \ldots, \sigma(m-1)$, each with $d$ consecutive values.

For the typical case of a tetrahedral mesh, twelve values are loaded per $T$, because each cell has four vertices, and each vertex has three coordinates. The code in Algorithm 2 shows how the vertex coordinates of a cell may be gathered in this case. Note that the restrict keyword is used as a hint to the compiler that accesses through the different pointers will not refer to the same memory locations, thereby allowing the compiler to apply certain optimisations that it otherwise might not. 


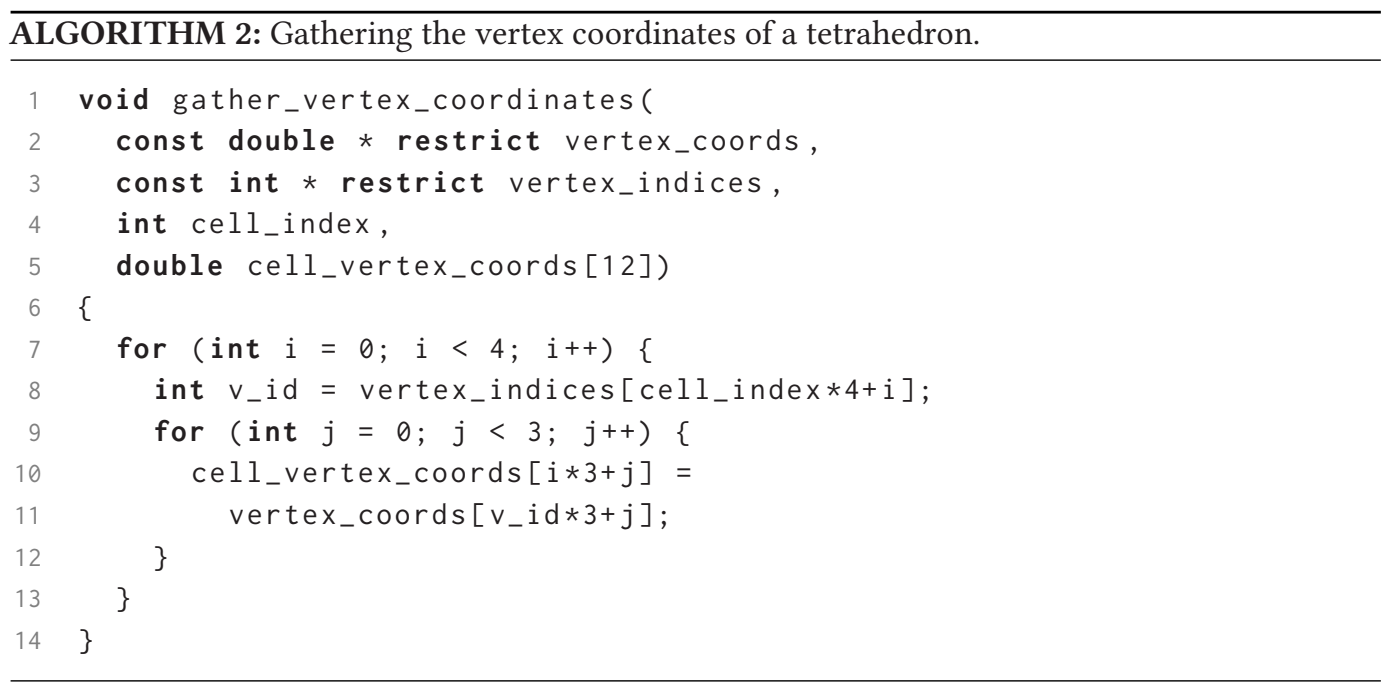

\subsection{Transforming to a Reference Cell}

Since the element matrices are computed by integration, it is customary to first perform a change of variables that shifts the domain of integration from a given cell $T \in \mathcal{T}$ to a reference cell $\hat{T} \subset \mathbf{R}^{d}$. The change of variables is needed to apply standard numerical integration schemes, which are defined for integrals over certain reference domains, such as the standard simplex or unit cube. In addition, some parts of the integrals can often be simplified or precomputed, because they are independent of the mesh geometry.

Given a mesh cell $T \in \mathcal{T}$ and a reference cell $\hat{T} \subset \mathbf{R}^{d}$, let $F: \hat{T} \rightarrow T$ be a one-to-one $C^{1}$-mapping, such that $F^{\prime}(\hat{x}) \neq 0$ for all $\hat{x} \in \hat{T}$, where $F^{\prime}(\hat{x})$ is the Jacobian of $F$, i.e., $\left(F^{\prime}(\hat{x})\right)_{i, j}=\frac{\partial F_{i}}{\partial \hat{x}_{j}}(\hat{x})$. In general, for a continuous function $f: \mathbf{R}^{d} \rightarrow \mathbf{R}$, whose support is compact and lies in $T$, a change of variables from $T$ to a reference cell $\hat{T}$ gives

$$
\int_{T} f(x) \mathrm{d} x=\int_{\hat{T}} f(F(\hat{x}))\left|\operatorname{det} F^{\prime}(\hat{x})\right| \mathrm{d} \hat{x} .
$$

In the case of a $d$-dimensional simplicial mesh, the reference cell is usually the standard simplex, $\hat{T}=\left[e_{0} e_{1} \cdots e_{d-1} \mathbf{0}\right]$, where $e_{i} \in \mathbf{R}^{d}$ is the $i$ th unit vector, $0 \leq i<d$, and $\mathbf{0} \in \mathbf{R}^{d}$ is the zero vector. If $T$ is a $d$-dimensional simplex with vertices $p_{0}, p_{1}, \ldots, p_{d} \in \mathbf{R}^{d}$, then an affine mapping from the standard simplex to $T$ is defined by

$$
F(\hat{x})=p_{d}+\sum_{k=0}^{d-1} \hat{x}_{k}\left(p_{k}-p_{d}\right) .
$$

The Jacobian of this mapping is

$$
\left(F^{\prime}(\hat{x})\right)_{i, j}=\left(p_{j}-p_{d}\right) \cdot e_{i}=p_{j, i}-p_{d, i},
$$

which, it may be noted, is constant over $\hat{T}$ and does not depend on $\hat{x}$. Furthermore, the inverse of the Jacobian $\left(F^{\prime}(\hat{x})\right)^{-1}$ is often required and can be computed from Cramer's rule,

$$
\left(F^{\prime}(\hat{x})\right)^{-1}=\frac{1}{\operatorname{det} F^{\prime}(\hat{x})} C_{F^{\prime}}^{\top}
$$


where $C_{F^{\prime}}$ is the matrix of cofactors of $F^{\prime}(\hat{x})$. Recall that the $(i, j)$ th cofactor of a matrix is computed from the determinant of the sub-matrix obtained by omitting the $i$ th row and $j$ th column and multiplying by a factor $(-1)^{i+j}$.

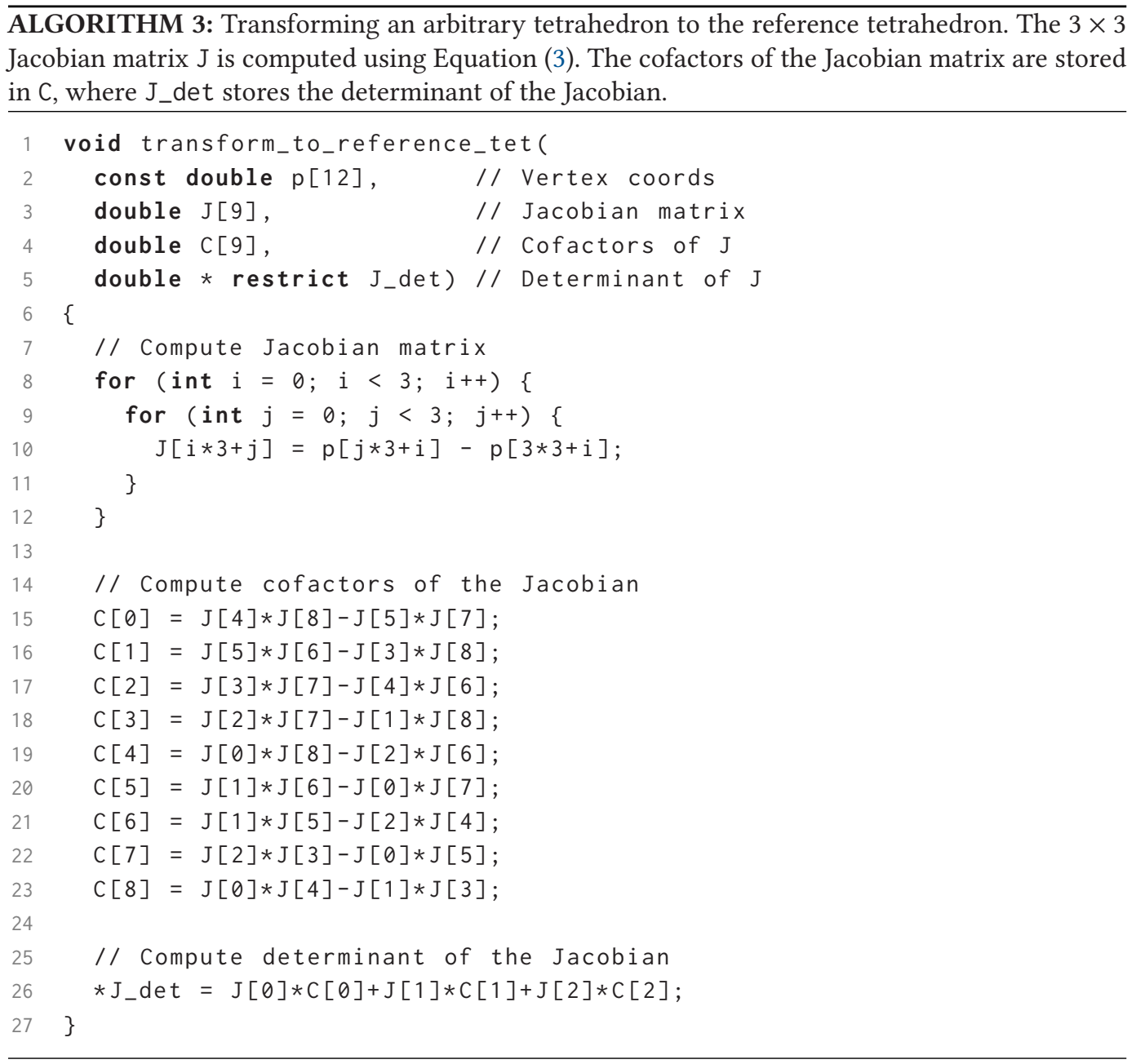

For a tetrahedral mesh, the code in Algorithm 3 shows how the transformation to the reference tetrahedron is computed, requiring a total of 41 floating point operations.

\subsection{Computing the Element Matrix}

Once a cell's vertex coordinates and the mapping to a reference cell have been obtained, the element matrix can be computed. This kernel must be tailored to an individual variational form. Alternatively, projects such as FEniCS [Logg et al. 2012] and Firedrake [Rathgeber et al. 2016] can ease the burden of hand-crafting these kernels by automatically generating code that will compute element matrices based on high-level descriptions of variational forms. 
3.3.1 Laplacian. To illustrate, let us first consider the variational form for the Laplacian with respect to a test space $V(\mathcal{T})$ and trial space $U(\mathcal{T})$,

$$
a(v, u)=\int_{\Omega} \nabla v \cdot \nabla u \mathrm{~d} x .
$$

In this example, the element matrix $A_{T}$ is

$$
\left(A_{T}\right)_{i, j}=\int_{T} \nabla \psi_{i} \cdot \nabla \phi_{j} \mathrm{~d} x
$$

for local basis functions $\psi_{i} \in V(T)$ and $\phi_{j} \in U(T)$. Applying a change of variables, based on a mapping $F: \hat{T} \rightarrow T$ for a reference cell $\hat{T}$, leads to

$$
\left(A_{T}\right)_{i, j}=\int_{\hat{T}}\left(F^{\prime}(\hat{x})\right)^{-\mathrm{T}} \nabla \hat{\psi}_{i} \cdot\left(F^{\prime}(\hat{x})\right)^{-\mathrm{T}} \nabla \hat{\phi}_{j}\left|\operatorname{det} F^{\prime}(\hat{x})\right| \mathrm{d} \hat{x},
$$

where $\hat{\psi}_{i}=\psi_{i} \circ F$ and $\hat{\phi}_{j}=\phi_{j} \circ F$. Note that $\hat{\psi}_{i}$ and $\hat{\phi}_{j}$ are local basis functions for $V(\hat{T})$ and $U(\hat{T})$, respectively.

Integrals are usually computed by using a numerical integration scheme, whereby the integrand is evaluated at a number of quadrature points, multiplied by suitable weights, and then the results are added together. However, element matrices can sometimes be rewritten into a tensor representation [Kirby and Logg 2006] by factoring out terms that are independent of the cell geometry. More specifically, the element matrix is written as a tensor contraction $A_{T}=\sum_{\alpha} \hat{A}_{\alpha} G_{T}^{\alpha}$, where $\hat{A}$ is a reference tensor and $G_{T}$ is a geometry tensor that depends on the mesh cell $T$. In the case of an affine mesh and a variational form with constant coefficients, the integrals appearing in the reference tensor can be precomputed and there is no need to perform any numerical integration at runtime. This strategy is employed by FEniCS in its automated generation of kernels for computing element matrices.

For example, the element matrix for the Laplacian can be written as the contraction of a reference tensor $\hat{A}$ of rank four and a geometry tensor $G_{T}$ of rank two. More specifically,

$$
\hat{A}_{i, j, k_{0}, k_{1}}=\int_{\hat{T}} \frac{\partial \hat{\psi}_{i}}{\partial \hat{x}_{k_{0}}} \frac{\partial \hat{\phi}_{j}}{\partial \hat{x}_{k_{1}}} \mathrm{~d} \hat{x},
$$

for $0 \leq i<\operatorname{dim} V(\hat{T}), 0 \leq j<\operatorname{dim} U(\hat{T})$, and

$$
G_{T}^{k_{0}, k_{1}}=\frac{1}{\left|\operatorname{det} F^{\prime}\right|} \sum_{l=0}^{d-1}\left(C_{F^{\prime}}\right)_{l, k_{0}}\left(C_{F^{\prime}}\right)_{l, k_{1}},
$$

for $0 \leq k_{0}, k_{1}<d$. Because the basis functions with respect to the reference element are known beforehand, the reference tensor can be precomputed. Algorithm 4 shows a kernel that uses this approach to compute element matrices for the Laplacian with first-order Lagrange elements on tetrahedra. The kernel for second-order elements is similar, though additional basis functions result in a $10 \times 10$ element matrix.

3.3.2 Laplacian with a Variable Coefficient. Next, we consider how to form element matrices for the Laplacian with a variable coefficient $c: \Omega \rightarrow \mathrm{R}$, for which the variational form is

$$
a(v, u ; c)=\int_{\Omega} c \nabla v \cdot \nabla u \mathrm{~d} x .
$$

The element matrix is given by

$$
\left(A_{T}\right)_{i, j}=\int_{\hat{T}} c(F(\hat{x}))\left(F^{\prime}(\hat{x})\right)^{-\mathrm{T}} \nabla \hat{\psi}_{i} \cdot\left(F^{\prime}(\hat{x})\right)^{-\mathrm{T}} \nabla \hat{\phi}_{j}\left|\operatorname{det} F^{\prime}(\hat{x})\right| \mathrm{d} \hat{x} .
$$




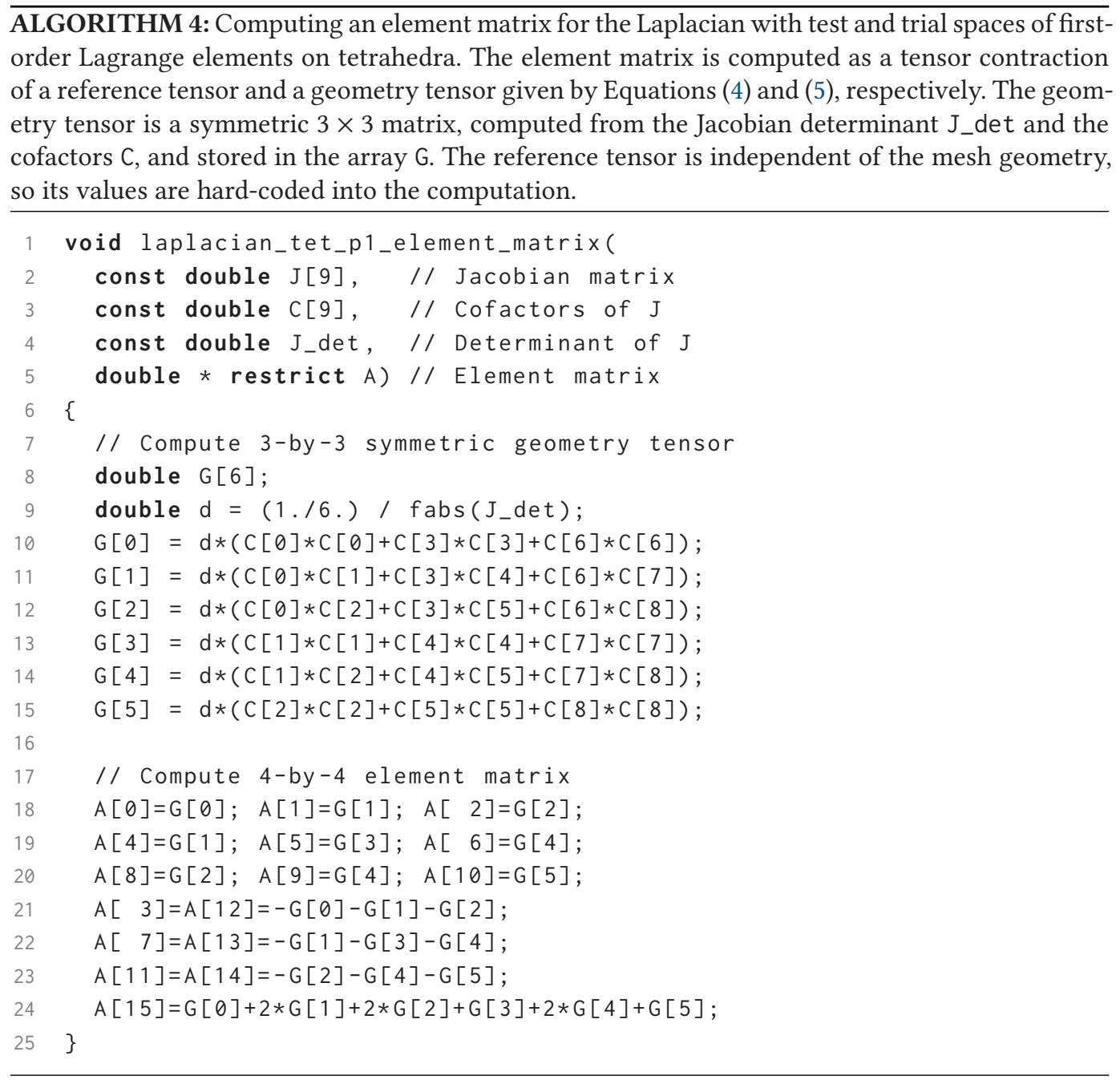

Here, we examine two cases that differ in how the coefficient is specified. Once again, we assume that the mesh is affine and derive the tensor representations of the element matrices.

In the first case, the coefficient $c$ is given by some analytic expression of the spatial coordinates $x_{0}, x_{1}, \ldots, x_{d-1}$. Though it is sometimes possible to compute the integrals in Equation (7) exactly, it is more often the case that a numerical quadrature scheme is applied. That is, $\int_{\hat{T}} f(\hat{x}) \mathrm{d} \hat{x} \approx$ $\sum_{k} \omega_{k} f\left(\xi_{k}\right)$, where $\omega_{k}$ and $\xi_{k}$ are weights and quadrature points. This leads to a geometry tensor

$$
G_{T}^{k_{0}, k_{1}, k_{2}}=\frac{c\left(F\left(\xi_{k_{2}}\right)\right)}{\left|\operatorname{det} F^{\prime}\right|} \sum_{l=0}^{d-1}\left(C_{F^{\prime}}\right)_{l, k_{0}}\left(C_{F^{\prime}}\right)_{l, k_{1}}
$$

and reference tensor

$$
\hat{A}_{i, j, k_{0}, k_{1}, k_{2}}=\omega_{k_{2}} \frac{\partial \hat{\psi}_{i}}{\partial \hat{x}_{k_{0}}}\left(\xi_{k_{2}}\right) \frac{\partial \hat{\phi}_{j}}{\partial \hat{x}_{k_{1}}}\left(\xi_{k_{2}}\right),
$$

for $0 \leq k_{0}, k_{1}<d$ and $0 \leq k_{2}<K$, where $K$ is the number of quadrature points. 
In the second case, there is no known analytic expression for the coefficient $c$, but it is instead given as a function belonging to a finite element space $W(\mathcal{T})$. For example, if $c$ is piecewise linear and its values are known at the mesh vertices, then it can be represented in terms of first-order Lagrange elements. In any event, within a given cell, $x \in T$, we can be expand $c$ into a sum $c(x)=\sum_{k} c_{\zeta(k)} \theta_{k}(x)$, where $\zeta$ denotes the local-to-global mapping, $\theta_{k}$ is a local basis function on $W(T)$, and $c_{\zeta(k)}$ is the coefficient of the corresponding global basis function. Inserting into Equation (7), we find that the geometry and reference tensors now become

$$
G_{T}^{k_{0}, k_{1}, k_{2}}=\frac{c_{\zeta\left(k_{2}\right)}}{\left|\operatorname{det} F^{\prime}\right|} \sum_{l=0}^{d-1}\left(C_{F^{\prime}}\right)_{l, k_{0}}\left(C_{F^{\prime}}\right)_{l, k_{1}},
$$

and

$$
\hat{A}_{i, j, k_{0}, k_{1}, k_{2}}=\int_{\hat{T}} \hat{\theta}_{k_{2}} \frac{\partial \hat{\psi}_{i}}{\partial \hat{x}_{k_{0}}} \frac{\partial \hat{\phi}_{j}}{\partial \hat{x}_{k_{1}}} \mathrm{~d} \hat{x}
$$

for $0 \leq k_{0}, k_{1}<d$ and $0 \leq k_{2}<\operatorname{dim} W(T)$. The integrals appearing in the reference tensor can be precomputed exactly, in the same way as for the constant-coefficient case.

\subsection{Scattering Results to a Global Matrix}

In the final stage of the cellwise assembly, the contributions from an element matrix $A_{T}$ are added to the global matrix $A$. Let $V(T)$ and $U(T)$ denote local finite element spaces with local-to-global mappings $\mu:[0, m) \rightarrow[0, M)$ and $v:[0, n) \rightarrow[0, N)$, respectively. Then, for each pair of local basis functions, $\left(\psi_{i}, \phi_{j}\right)$, for $0 \leq i<m$ and $0 \leq j<n$, the corresponding value of the global matrix is updated,

$$
A_{\mu(i), v(j)} \leftarrow A_{\mu(i), v(j)}+\left(A_{T}\right)_{i, j}
$$

However, because the global matrix $A$ is sparse with only its non-zeros stored, it is necessary to locate the correct position in the array that stores the non-zero values of $A$ before each update.

We assume that the global matrix $A$ is stored in the commonly used format of compressed sparse row (CSR). Suppose there are in total $K$ non-zero entries $a_{0}, a_{1}, \ldots, a_{K-1}$, arranged in ascending order according to their row and column indices $\left(i_{k}, j_{k}\right), 0 \leq k<K$. Then, in addition to the $K$ nonzeros, we store the $K$ column indices $j_{0}, j_{1}, \ldots, j_{K-1}$ and $(M+1)$ row pointers $r_{0}, r_{1}, \ldots, r_{M}$. The row pointers $r_{i}$ and $r_{i+1}-1$ are the indices of the first and last non-zeros of the $i$ th row, respectively. In other words, a non-zero $a_{k}$ belongs to the $i$ th row if $r_{i} \leq k<r_{i+1}$.

Now, it remains to find the non-zero matrix entry $a_{k}$ that corresponds to the global matrix value $A_{\mu(i), v(j)}$. For a CSR matrix, the row pointers are used to find matrix entries that belong to the given row, i.e., $r_{\mu(i)} \leq k<r_{\mu(i)+1}$. Because the non-zeros within each row are sorted by their column indices, a common strategy is to perform a binary search to find the non-zero matrix entry $a_{k}$ whose column index equals $v(j)$. Algorithm 5 shows how this is done when the test and trial spaces are first-order Lagrange elements on tetrahedra.

\subsection{Parallelisation}

Parallel algorithms are needed to boost performance and enable large-scale problems to be solved. Though many finite element codes rely on distributed-memory parallel techniques, we limit our attention to shared-memory, multi-core systems. Obtaining good performance on a single multicore system is not only challenging in itself, but it also lays a solid foundation for later scaling to multiple compute nodes with distributed memory. Here we consider the case of assembling a global matrix, though the following applies equally to the case of assembling a vector.

For cellwise finite element assembly (see Algorithm 1), there is a commonly used, straightforward way of parallelisation for multiple threads with shared memory. This is done by assigning 


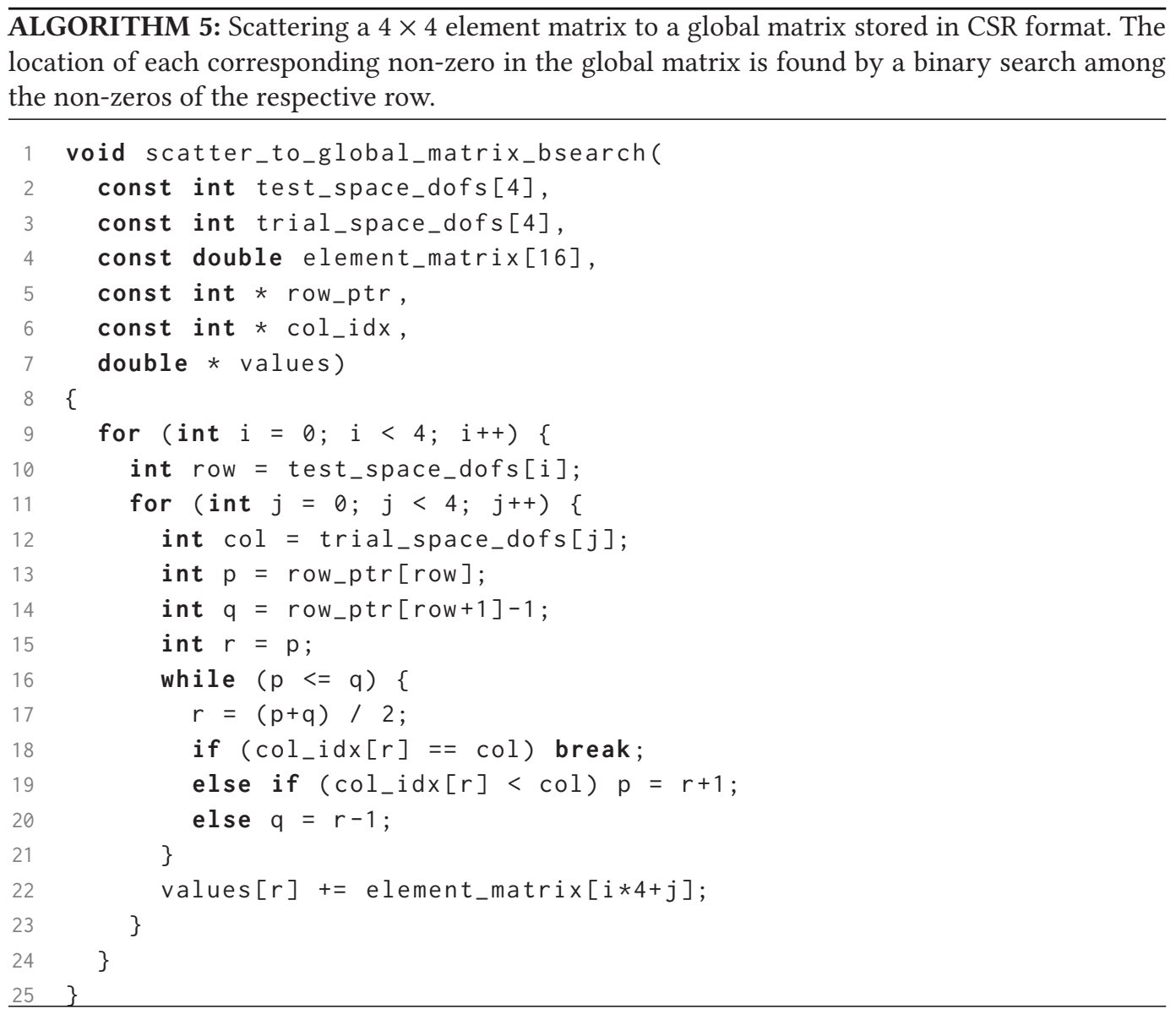

a roughly equal number of cells to each thread, and then letting each thread independently compute element matrices for the mesh cells it has been assigned. Each thread must also scatter its element matrices to the global matrix. However, the global matrix is shared among threads, which leads to different threads updating the same locations in the global matrix. This occurs whenever neighbouring mesh cells are assigned to different threads and some global basis functions belong to more than one mesh cell. These shared, global basis functions correspond directly to rows and columns of the global matrix whose values must be updated by multiple threads.

To avoid incorrect results, one must take care to prevent race conditions from updating shared values in the global matrix. This is typically achieved by introducing some kind of synchronisation between threads. One strategy is to colour mesh cells so that neighbouring cells have different colours, then perform assembly one colour at a time with synchronisation points after each colour has been processed (see, e.g., Kronbichler and Kormann [2012] and Markall et al. [2013]). In this article, we prefer instead to use atomic operations to update shared values and avoid race conditions, which has the advantage of being particularly easy to implement. Moreover, there is no need to preprocess the mesh to find a colouring, and we avoid a potential loss of data locality, since mesh cells can be processed in any order rather than being treated one colour at a time.

Here we use OpenMP to implement a shared-memory parallel version of the cellwise finite element assembly. This amounts to adding a \#pragma omp parallel for the directive to parallelise 
the outer loop over the mesh cells, as well as a \#pragma omp atomic directive above line 22 in Algorithm 5, where values of the global matrix are updated.

\section{OPTIMISATIONS}

In this section, we consider some optimisations that are relevant for the finite element assembly algorithm described in Section 3. Note that most of the optimisations considered here, including data reordering, the use of a lookup table, as well as row-wise assembly, incur some initial cost due to, for example, setting up data structures that are needed. However, it is often the case-for instance, due to solving a nonlinear or time-dependent problem-that the additional setup cost is amortised by assembling multiple matrices using the same variational form, mesh and matrix sparsity pattern.

\subsection{Reordering Unstructured Meshes}

The arithmetic intensity of finite element assembly algorithms is low when low-order Lagrange elements are used. Therefore, it is prudent to consider optimisations that improve data locality and use caches more effectively.

The memory access patterns and data locality associated with gathering the vertex coordinates of each mesh cell (see Algorithm 2) depends on the order in which mesh cells are visited. Therefore, reordering the vertices and cells of an unstructured mesh, prior to the finite element assembly, can yield improved performance. For similar purposes, researchers have previously studied cache-efficient mesh layouts, for instance, to improve the performance of visualising unstructured meshes [Tchiboukdjian et al. 2008, 2010; Yoon and Lindstrom 2006].

In this article, we opt for a simple approach that is commonly used in connection with finite element methods. First, we reorder the vertices of a mesh based on the Reverse Cuthill-McKee (RCM) algorithm [Cuthill and McKee 1969]. Specifically, we apply the RCM algorithm to an adjacency matrix whose rows and columns correspond to the mesh vertices and whose entries indicate whether or not a pair of vertices has a mesh cell in common. Since the RCM algorithm is designed to reduce the bandwidth of a sparse matrix, then, roughly speaking, this method moves vertices closer to each other whenever they belong to the same mesh cells. A potential limitation of this approach is that matrix bandwidth is only indirectly related to data locality. That being said, RCM turns out to be quite effective in practice, as we will see later in Section 6.

It is important to also reorder the cells of a mesh, because a good vertex ordering alone does not imply good data locality when gathering the vertex coordinates of each mesh cell. Here we adopt a simple technique, which, as shown in Section 6, appears to be quite effective in practice. That is, we rearrange the mesh cells in (ascending) lexicographic order according to their vertex indices. More specifically, consider a mesh cell $T_{i}$, given by $m$ distinct vertices $\sigma_{i}(0), \sigma_{i}(1), \ldots, \sigma_{i}(m-1)$, and another mesh cell $T_{j}$ similarly defined by the vertices $\sigma_{j}(0), \sigma_{j}(1), \ldots, \sigma_{j}(m-1)$. We sort the cells so that $T_{i}<T_{j}$ if there is a $k, 0 \leq k<m$, such that $\sigma_{i}(l)=\sigma_{j}(l)$ for $0 \leq l<k$ and $\sigma_{i}(k)<\sigma_{j}(k)$. The same approach is also used to define an ordering of other mesh entities, such as edges and faces.

Note that we have also chosen to first reorder the vertex indices of each individual cell, so that the vertex indices appear in increasing order. In other words, we have that $\sigma_{i}(0)<\sigma_{i}(1)<\cdots<$ $\sigma_{i}(m-1)$ for the vertex indices of a mesh cell $T_{i}$. This is not strictly necessary, and may not be desirable if the mesh orientation is important, since the orientation of each cell must then be stored explicitly. However, there is a good chance that ordering each cell's vertices in this manner further improves data locality. 


\subsection{Reordering Global Degrees of Freedom}

When scattering element matrices to a global matrix in the CSR format (see Algorithm 5), the numbering of the global degrees of freedom greatly impacts the memory access pattern and data locality. Generally speaking, reordering the global degrees of freedom of the test and trial spaces is equivalent to reordering the equations and unknowns of the assembled linear system, and this is a well-known method for improving the performance of sparse linear solvers, particularly direct solvers. In fact, there exists a variety of sparse matrix reorderings that are commonly used in connection with finite element methods [George 1973; George and Mcintyre 1978], including the RCM algorithm [Cuthill and McKee 1969] that we already use to reorder the mesh vertices.

For first-order Lagrange elements, the global degrees of freedom are usually numbered in the same way as the mesh vertices. Thus, reordering the mesh vertices is equivalent to reordering the global degrees of freedom in this case. For second- and higher-order elements, it is also possible to apply the RCM algorithm to the assembled matrix, which typically results in an ordering that interleaves global degrees of freedom associated with mesh entities of different kinds. However, in this article, we consider another approach, where mesh entities are first ordered by dimensionthat is, vertices, edges, faces, then cells-and global degrees of freedom are thereafter ordered according to the mesh entities that they belong to. Recall that we already arrange for mesh entities of a given dimension to appear in lexicographic order according to their vertex indices. In this way, we have chosen an ordering of the mesh entities, and the global degrees of freedom will be numbered accordingly.

\subsection{Using a Lookup Table in the Scattering Kernel}

Another concern that affects the performance of scattering element matrices to a global matrix is the binary search (Algorithm 5) that is performed for each entry of an element matrix. This is needed to find the location within the CSR structure of the corresponding non-zero entry in the global matrix. As an alternative, it is possible to precompute and store the locations of the global matrix non-zeros for each entry of every element matrix. The non-zero locations can usually be obtained for free or at little extra cost when the sparsity pattern of the global matrix is computed prior to the assembly. The lookup table can then be reused every time the matrix is assembled.

Although this approach requires a slight increase in storage requirements and memory traffic to accommodate the lookup table, the benefit is that the binary searches, which are potentially quite costly, can now be omitted. Moreover, it is no longer necessary to access the row pointers or column indices of the global matrix when scattering element matrices. This counteracts the added memory traffic from the lookup table and potentially reduces memory traffic overall. An example of this approach is shown in Algorithm 6, which should be contrasted with Algorithm 5.

\subsection{Cross-element Vectorisation}

Vectorisation is often needed to make the most effective use of multi-core CPUs, especially for kernels involving a lot of floating point calculations. Such techniques were used on vector processors [Carey et al. 1988] and are still used in contemporary finite element software [Fischer et al. 2020; Kronbichler and Kormann 2012; Moxey et al. 2020; Sun et al. 2020] to speed up the calculation of element matrices, usually based on a technique known as cross-element vectorisation. Although compilers are sometimes able to automatically perform vectorisation, there are cases where such optimisations must be applied manually. We note that the kernels we consider here are fairly lightweight in terms of floating point calculations, so we do not expect vectorisation to have too much of an impact on the overall assembly time. But we include it nonetheless for the sake of completeness, and because it may play a more important role for more advanced kernels. 


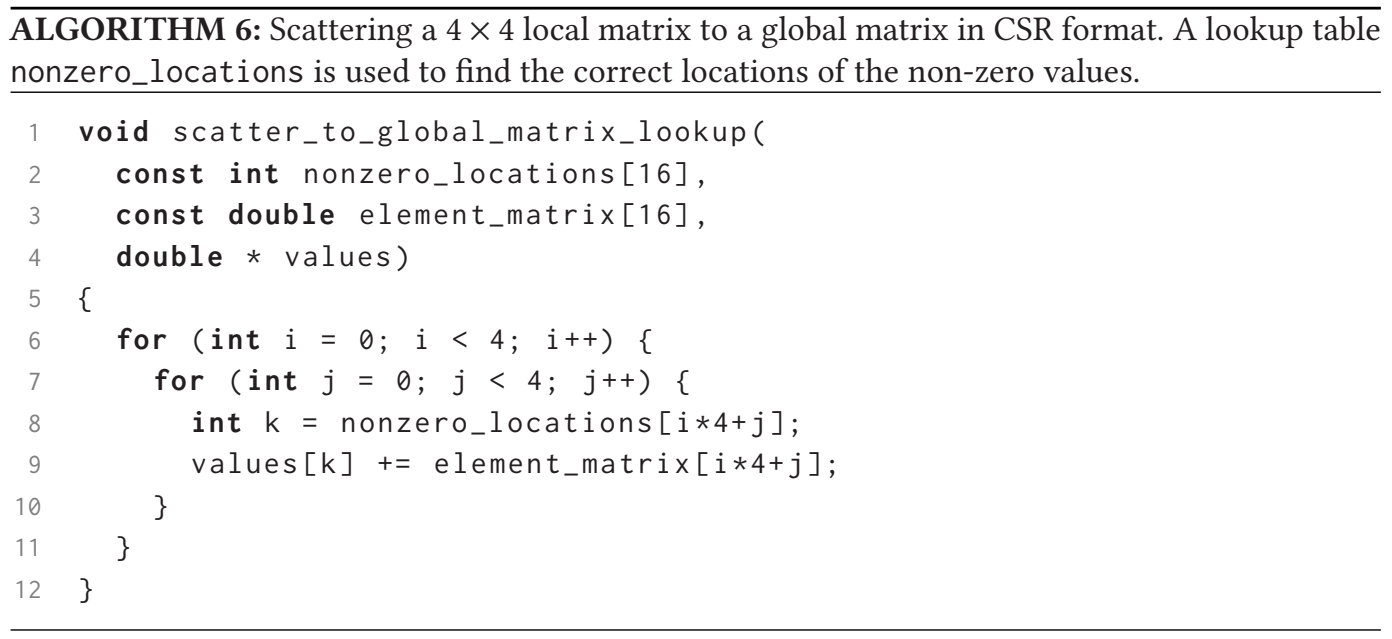

The idea behind cross-element vectorisation is to compute element matrices of several cells simultaneously by letting each vector lane correspond to a different mesh cell. This requires rearranging the input data-i.e., the vertex coordinates of the cells-after being brought into the firstlevel cache. Vector operations are primarily designed to act on a sequence of contiguous memory locations, and the vertex coordinates of each cell must therefore be interleaved.

Once the input data has been rearranged, the element matrix computation becomes similar to the scalar kernel. In particular, using specialised data types for vector instruction sets, such as AVX-512, and operator overloading for standard arithmetic operations, the code for computing element matrices for the Laplacian using cross-element vectorisation looks almost identical to that of Algorithm 4. The main difference is that the data type double is replaced with __m512d, which represents a vector of eight double-precision floating point values, and the intrinsic _mm512_abs_pd is used instead of fabs.

\subsection{Row-wise Assembly}

Recall that the most common strategy for parallelising a cellwise finite element assembly in shared memory, as described in Section 3.5, involves partitioning cells among threads and using, for example, mesh colouring or atomic operations to avoid race conditions when scattering element matrices to the global matrix. An alternative to the usual cellwise assembly algorithm is to compute an entire row of the global matrix at a time. From Equation (1), we find that the $i$ th row and $j$ th column of the global matrix $A$ can be expressed

$$
A_{i, j}=\sum_{T \in \mathcal{T}}\left(P_{T} A_{T} Q_{T}^{\top}\right)_{i, j}=\sum_{T \in \mathcal{T}} \sum_{k=0}^{m-1} \delta_{i, \mu(k)}\left(A_{T} Q_{T}^{\top}\right)_{k, j},
$$

where $\mu$ is a local-to-global mapping for the test space $V(\mathcal{T})$. Recall that the $i$ th row of the matrix corresponds to a global basis function $\theta_{i} \in V(\mathcal{T})$, and this global basis function is associated with some mesh entity $f_{i}-$ a vertex, edge, face or the interior of a cell-of the mesh $\mathcal{T}$. Moreover, for a mesh cell $T$, we have $\delta_{i, \mu(k)} \neq 0$ only if $f_{i} \subseteq T$. Therefore, the above sum can be restricted to those cells that contain the mesh entity $f_{i}$, so that

$$
A_{i, j}=\sum_{\substack{T \in \mathcal{T} \\ f_{i} \subseteq T}}\left(A_{T} Q_{T}^{\top}\right)_{\eta(i), j}
$$


where $\eta:[0, M) \rightarrow[0, m)$ is a left inverse of $\mu$. That is, $\eta$ maps global basis functions of the test space $V(\mathcal{T})$ to basis functions of the local space $V(T)$.

Ultimately, the above may be translated to the high-level pseudo-code for a row-wise assembly algorithm that is shown in Algorithm 7.

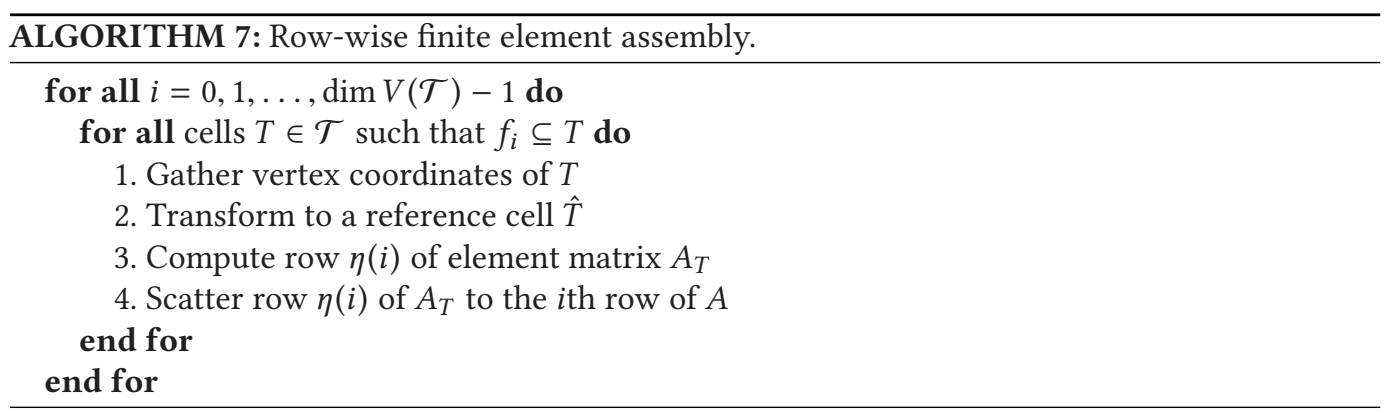

The basic building blocks of the above algorithm are the same as those used in the cellwise assembly, but there are some differences. Rather than computing and scattering the entire element matrix in steps 3 and 4, only a single row is needed in each iteration of the inner loop. Therefore, the order in which the element matrices are computed and scattered is different compared to a cellwise assembly, but the total amount of work carried out during these two steps remains the same. On the other hand, the first two steps, gathering a cell's vertex coordinates and transforming to a reference cell, are now carried out more often than before. For example, for a tetrahedral mesh and first-order Lagrange elements, these steps occur exactly four times more often than in a cellwise assembly. That is, each cell consists of four vertices and steps 1 and 2 are executed every time a cell contains the vertex corresponding to the current row of the global matrix.

If we compare the cellwise and row-wise assembly algorithms, then the latter performs more work. However, the memory access pattern of the row-wise assembly is more regular because rows of the global matrix are accessed in sequence, and only a single row is written during an iteration of the outer loop. This is in stark contrast to the irregular memory access pattern of cellwise assembly, which jumps back and forth between rows of the global matrix that are potentially far apart. Thus, the row-wise assembly algorithm presents an interesting tradeoff by needing some redundant computations as well as extra memory traffic to gather vertex coordinates, but at the same time generating less memory traffic to write the results to the global matrix due to improved data locality. More importantly, the row-wise algorithm can easily be parallelised without introducing any synchronisation overhead to avoid race conditions. This is achieved by simply assigning different rows of the global matrix to different threads.

\section{MEMORY TRAFFIC ESTIMATES}

In this section, we derive some lower and upper bounds of the memory traffic that are expected for the finite element assembly kernels from Section 3. These estimates are useful for evaluating the impact of memory traffic on different kernels and the effectiveness of some of the optimisations from Section 4.

We are concerned with multi-core CPU systems with one or more sockets that each contains multiple cores. Each CPU core is equipped with one or more floating point functional units (FPUs) that perform floating point calculations on operands that are supplied from the CPU core's working memory, or registers. Data that is needed for computation, but is not already located in registers, must be fetched from memory. Conversely, intermediate results that do not fit in registers, 


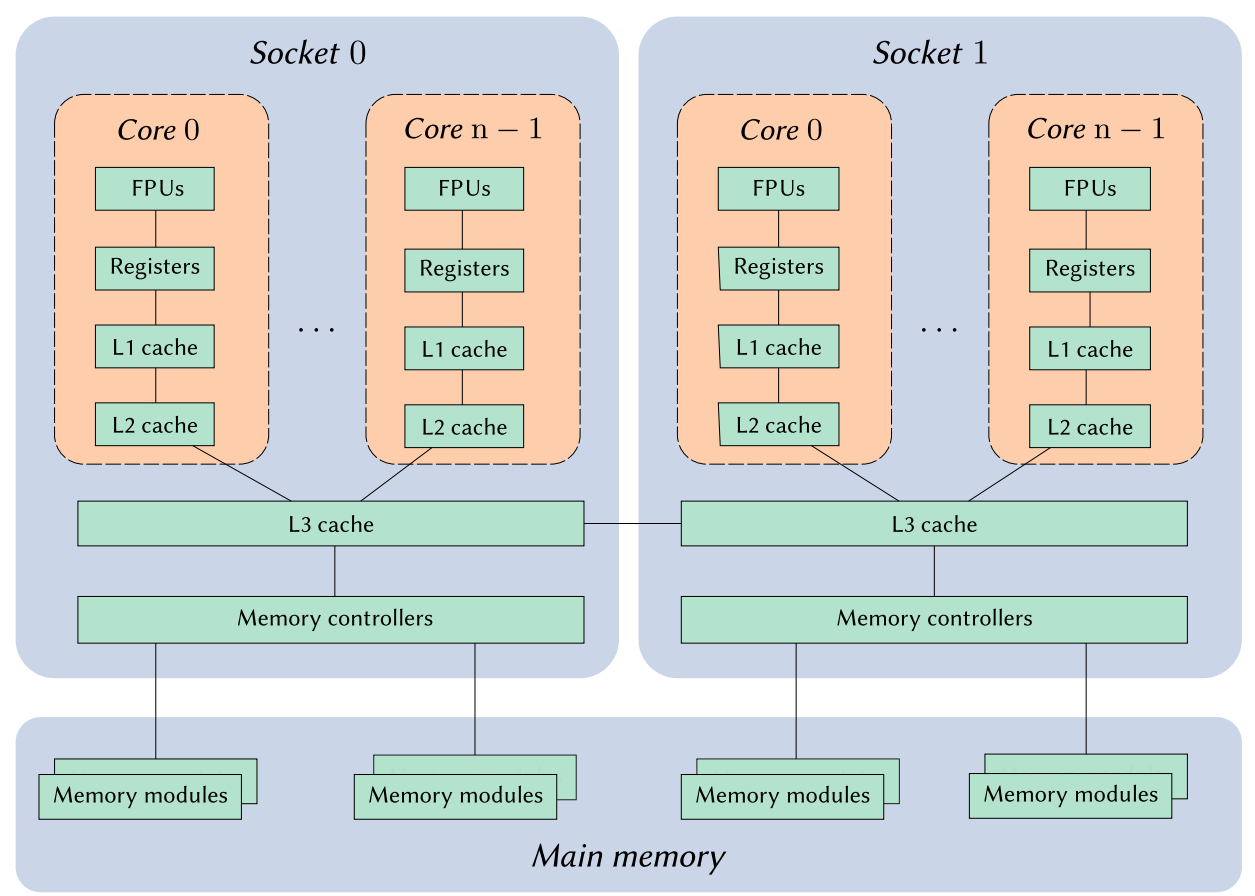

Fig. 1. A simplified diagram of the architecture of a two-socket, multi-core CPU system and its memory hierarchy. Each core has one or more FPUs, a working memory, also called registers, and two levels of caches. A third-level cache is most often shared by all the cores of a socket. Finally, each socket has one or more memory controllers that are used to transfer data between third-level caches and main memory.

or results that are otherwise to be saved for later, must be written to memory. Moreover, memory is organised in a multi-level hierarchy, as depicted in Figure 1. At the highest level of a typical memory hierarchy, that is, closest to the CPU registers, each core has a smaller, first-level (L1) cache and a larger second-level (L2) cache. Next, a third-level (L3) cache is usually shared by some or all of the cores on a given socket. Finally, main memory, or DRAM, occupies the lowest level of the memory hierarchy. As a general rule, higher-level memories are faster but have smaller storage capacities than lower-level ones.

\subsection{Ideal Cache Model}

For modelling purposes, it is often convenient to assume the ideal cache model [Frigo et al. 2012]. In this model, a cache is characterised by two properties. First, the cache size, $Z$, is the number of words (numerical values) that can be held in the cache at any time. Second, the main memory is partitioned into equally sized blocks, called cache blocks. The size of these blocks is referred to as the cache line size, $L$, the number of words held per block. The effect is that entire cache blocks are transferred, rather than individual values, whenever data is moved between cache and main memory. This allows exploiting spatial locality by presuming that nearby memory locations are likely to soon be accessed or written to.

The cache model also determines when and where cache blocks are placed in a cache. First, caches are assumed to operate with demand caching, which means that a cache block is placed into a cache only if a CPU issues a load or store that references a memory location within the cache block. Second, we assume that caches are fully associative, which entails that a cache block can be placed anywhere in a cache. That is, unlike set-associative or direct-mapped caches, there 
are no restrictions that make only one or a few cache lines available for placing a given cache block. As a consequence, the ideal cache model ignores conflict misses, or cache misses that would occur due to cache blocks being mapped to the same set of cache lines in a set-associative or directmapped cache. Third, if the cache is full, then a cache line must be evicted, and its contents are written back to the main memory, to make room for an incoming cache block. In this case, the ideal cache model employs an optimal replacement policy, which evicts cache lines in a way that minimises the number of transfers between the cache and main memory.

\subsection{Gathering Vertex Coordinates}

To gather the vertex coordinates of each mesh cell, the data must be brought from whichever level of the memory hierarchy where it resides to the processor's registers. In the following, the cache line size $L$ denotes the number of double-precision floating point values that fit in a cache block, and we assume that $2 L$ integers fit in the same cache block. The most common cache line size is 64 bytes, such that $L=8$. Furthermore, let $w$ denote the vector width, which is the number of consecutive double precision floating point values that may be read or written with a single load or store. Similarly, we assume that twice the vector width, $2 w$, applies when working with integers. In practice, the most relevant cases are $w=1,2,4$, or 8 .

First, note that the vertex indices are accessed sequentially. Unless the data resides in cache before the computation begins, there is no reuse of cached values. Therefore, in total $N \times\lceil\mathrm{m} /(2 \mathrm{w})\rceil$ loads are issued, where $N$ is the number of mesh cells and $m$ is the number of vertices in each cell. Moreover, $\lceil\mathrm{Nm} /(2 \mathrm{~L})\rceil$ cache blocks are transferred from lower levels of the memory hierarchy.

Second, reading the coordinates of a single vertex requires at least $\lceil d / w\rceil$ loads from the firstlevel cache, where $d$ is the number of coordinates for each vertex. If each vertex coordinate is brought to the cache exactly once due to perfect cache data reuse, then the total number of cache lines that are transferred is at least $\lceil M d / L\rceil$, where $M$ is the number of vertices in the mesh.

In the worst case, the coordinates of a vertex must be brought to the cache for every mesh cell that the vertex belongs to. If those coordinates are aligned to a cache line boundary, then they span $\lceil d / L\rceil$ cache lines. However, the vertex coordinates are usually not aligned to a cache line boundary, for instance, if $d=3$ for a tetrahedral mesh and cache lines may hold $L=8$ values. In this case, the coordinates of a vertex may span $\lceil d / L+1\rceil$ cache lines. Thus, the number of cache lines that are brought to the cache is at most $N m \times\lceil d / L+1\rceil$.

In summary, $N \times(\lceil m /(2 w)\rceil+m\lceil d / w\rceil)$ loads are issued, and the number of cache misses is at least $\lceil N m /(2 L)\rceil+\lceil M d / L\rceil$ and at most $\lceil N m /(2 L)\rceil+N m\lceil d / L+1\rceil$.

\subsection{Gathering Coefficients}

Note that in the case of computing an element matrix for the Laplacian with a variable coefficient, as in Equation (7), some additional values may need to be read from memory if the coefficient $c$ is given as a function in a finite element space $W(\mathcal{T})$. More specifically, to later compute the geometry tensor from Equation (10), we need the local-to-global mapping $\zeta(k)$ and the coefficient $c_{\zeta(k)}$ for $0 \leq k<\operatorname{dim} W(T)$. Estimating the memory traffic involved is dealt with in essentially the same way as the vertex coordinates. The local-to-global mapping is accessed sequentially, just like the vertex indices, but the coefficients are not. Unlike the vertex coordinates, there is no benefit from vectorisation when reading a single coefficient value per degree of freedom.

\subsection{Computing the Element Matrix}

Computing an element matrix consists mostly of arithmetic operations and there is little or no memory traffic involved. Input data is fetched beforehand when gathering vertex coordinates, and output values are written during the subsequent step when they are scattered to a global matrix. 
Some data may nonetheless be read from or written to at least the first-level cache, if the input or intermediate values cannot be held entirely in register memory. This is more likely to occur with larger element matrices, such as for second-order elements, which have more degrees of freedom present in a mesh cell.

\subsection{Scattering Results to a Global Matrix}

Recall that adding an element matrix value to a global matrix first requires a binary search or a table lookup before the relevant matrix value is updated. In either case, updating the global matrix values results in an irregular memory access pattern that is influenced by the order of the mesh cells as well as the numbering of the global basis functions. To scatter the values of an $m \times n$ element matrix means accessing $m$ different rows of the global matrix, and updating $n$ values in each row. Since the matrix values that need to be updated usually do not occupy contiguous memory locations, vectorisation is of little use. Therefore, the global matrix updates amount to $N \times m n$ loads and the same number of stores, where $N$ is the number of mesh cells. In the ideal case, where each global matrix value is brought to a cache exactly once, the number of cache blocks that must be transferred is at least $\lceil K / L\rceil$, where $K$ is the number of nonzero entries in the global matrix.

If a binary-search approach is used, then there are $2 \mathrm{Nm}$ loads issued to read row pointers and an average of $N m n \times \log _{2}(\bar{r})$ loads issued for reading column indices, where $\bar{r}=K / M$ is the average number of non-zeros per row and $M$ is the number of matrix rows. The row pointers and column indices also result in at least $\lceil(M+1) /(2 L)\rceil$ and $\lceil K /(2 L)\rceil$ additional cache blocks being transferred, respectively.

If a table lookup is used, as in Algorithm 6, then the row pointers and column indices are no longer needed. Instead, there are $N \times m n$ loads issued and $\lceil N m n /(2 L)\rceil$ cache blocks transferred for the lookup table. Despite the extra memory usage due to the lookup table, this approach results in fewer memory accesses overall and the accesses to the lookup table are regular and in sequence.

To summarise, $N \times m n$ stores are issued regardless. In addition, the binary-search approach requires $2 N m+N m n\left(1+\log _{2}(\bar{r})\right)$ loads, whereas the lookup-table approach requires $2 N m n$ loads. Also, the binary-search approach causes at least $\lceil K / L\rceil+\lceil(M+1) /(2 L)\rceil+\lceil K /(2 L)\rceil$ cache misses, whereas the lookup-table counterpart incurs at least $\lceil K / L\rceil+\lceil N m n /(2 L)\rceil$ cache misses.

\section{NUMERICAL EXPERIMENTS}

In this section, we describe numerical experiments that benchmark the performance of our finite element assembly algorithms. To understand the observed performance, we use measurements of memory traffic based on the hardware performance monitoring features of the CPUs and compare these with the memory traffic estimates from Section 5.

In each of the benchmarks described later in this section, the performance and memory traffic are reported as averages from ten trials. We have also verified that there is very little variation between trials. We do not explicitly clear any caches before or after a trial, but the data sets we have chosen are large enough compared to the relevant last-level caches, such that, in practice, no trial run will reuse cached data from a previous trial.

\subsection{Experimental Setup}

The following is a brief description of the hardware and parameters used in the subsequent numerical experiments.

6.1.1 Hardware. Most of the following experiments were conducted on a multi-core CPU system with two Intel Xeon Gold 6130 CPUs. In addition, we have used a dual-socket AMD Epyc 7601 system and a dual-socket Cavium ThunderX2 CN9980 system to study parallel performance and 
Table 1. Hardware Configurations

\begin{tabular}{|c|c|c|c|}
\hline & Intel Xeon Gold 6130 & AMD Ерус 7601 & Cavium TX2 CN9980 \\
\hline Instruction set & $x 86-64$ & $x 86-64$ & ARMv8.1 \\
\hline Microarchitecture & Skylake (server) & Zen & Vulcan \\
\hline Cores per socket & 16 & 32 & 32 \\
\hline Frequency & $1.9-3.6 \mathrm{GHz}$ & $2.7-3.2 \mathrm{GHz}$ & $2.0-2.5 \mathrm{GHz}$ \\
\hline \multicolumn{4}{|l|}{$\begin{array}{l}\text { Double precision } \\
\text { floating point perf. }\end{array}$} \\
\hline single core & 112 Gflop/s & $25.6 \mathrm{Gflop} / \mathrm{s}$ & $20 \mathrm{Gflop} / \mathrm{s}$ \\
\hline single socket & $972.8 \mathrm{Gflop} / \mathrm{s}$ & $691.2 \mathrm{Gflop} / \mathrm{s}$ & 512 Gflop/s \\
\hline \multicolumn{4}{|l|}{ Memory per socket } \\
\hline L3 cache & $22 \mathrm{MiB}$ & $64 \mathrm{MiB}$ & $32 \mathrm{MiB}$ \\
\hline DRAM channels & 6 & 8 & 8 \\
\hline DRAM transfer rate & $2.67 \mathrm{GHz}$ & $2.67 \mathrm{GHz}$ & $2.67 \mathrm{GHz}$ \\
\hline $\begin{array}{l}\text { Theoretical max. } \\
\text { bandwidth }\end{array}$ & $128 \mathrm{~GB} / \mathrm{s}$ & $171 \mathrm{~GB} / \mathrm{s}$ & $171 \mathrm{~GB} / \mathrm{s}$ \\
\hline \multicolumn{4}{|l|}{ STREAM bandwidth } \\
\hline Single core & $13.0 \mathrm{~GB} / \mathrm{s}$ & $18.6 \mathrm{~GB} / \mathrm{s}$ & $11.7 \mathrm{~GB} / \mathrm{s}$ \\
\hline Single socket & $74.4 \mathrm{~GB} / \mathrm{s}$ & $81.3 \mathrm{~GB} / \mathrm{s}$ & $111.3 \mathrm{~GB} / \mathrm{s}$ \\
\hline Dual socket & $147.1 \mathrm{~GB} / \mathrm{s}$ & $161.4 \mathrm{~GB} / \mathrm{s}$ & $221.3 \mathrm{~GB} / \mathrm{s}$ \\
\hline
\end{tabular}

scalability in Section 6.3. See Table 1 for a summary of the main characteristics of these systems. For further details on the CPUs, see Guo [2019], Intel Corporation [2018], and Schor [2018].

The Xeon Gold 6130 CPU is based on the Skylake server microarchitecture, and therefore it supports the AVX-512 instruction set, which is capable of performing 32 double-precision floatingpoint operations per cycle if vectorisation and fused multiply-add instructions (FMAs) are used. If a single core is used, the CPU frequency is $3.6 \mathrm{GHz}$ for scalar code and $3.5 \mathrm{GHz}$ for AVX512 , whereas if all sixteen cores are being used, the frequency is reduced to $2.8 \mathrm{GHz}$ and $1.9 \mathrm{GHz}$ for scalar and AVX-512 operations, respectively. In comparison, the AMD Epyc and Cavium TX2 CPUs can perform only eight double-precision floating-point operations per cycle. Despite having twice as many CPU cores and slightly higher clock speeds, the peak floating point performance of these CPUs is significantly lower than Intel Xeon.

Meanwhile, Intel Xeon has six memory channels, whereas AMD Epyc and Cavium TX2 have eight, which results in a higher memory bandwidth for the latter two systems. From the numbers measured by the Triad kernel of the STREAM bandwidth benchmark [McCalpin 2013], the realistic dual-socket memory bandwidth for the AMD Epyc system is about $10 \%$ higher than Intel Xeon, while the dual-socket memory bandwidth for Cavium TX2 is almost $40 \%$ higher than AMD Epyc.

The various benchmarks were compiled using GCC 9.2.0 with the options -03, -march=native, and -fopenmp.

6.1.2 Computational Meshes. In the following experiments, we use tetrahedral meshes from two different biomedical applications. The first set of meshes have previously been used in cardiac modelling [Marciniak et al. 2017], and they originate from patient data in a Danish study on cardiac disease [Jabbari et al. 2015]. The second set of meshes, which represent blood vessels with aneurysms, have been used in blood flow simulations, and they are based on data from the 
Table 2. Computational Meshes

\begin{tabular}{lrr}
\hline Mesh & \multicolumn{1}{l}{ Vertices } & \multicolumn{1}{l}{ Cells } \\
\hline Uniform mesh 1 & $1,771,561$ & $10,368,000$ \\
Uniform mesh 2 & $4,173,281$ & $24,576,000$ \\
Cardiac mesh 1 & $1,255,775$ & $6,735,654$ \\
Cardiac mesh 20 & $3,019,809$ & $16,907,270$ \\
Cardiac mesh 41 & $2,226,802$ & $12,255,517$ \\
Cardiac mesh 44 & $1,958,816$ & $10,697,116$ \\
Aneurysm mesh 3 & 855,668 & $5,173,053$ \\
Aneurysm mesh 4 & $1,923,234$ & $11,717,169$ \\
\hline
\end{tabular}

Table 3. Single-core Performance and Memory Traffic for Gathering Vertex Coordinates of Tetrahedral Meshes on Intel Xeon Gold 6130

\begin{tabular}{|c|c|c|c|c|c|c|c|c|}
\hline \multirow[b]{2}{*}{ Mesh } & \multicolumn{2}{|c|}{ Est. DRAM read [B/cell] } & \multicolumn{2}{|c|}{ Meas. DRAM read [B/cell] } & \multicolumn{2}{|c|}{ Page walks [walk/cell] } & \multicolumn{2}{|c|}{ Performance [Mcell/s] } \\
\hline & Best case & Worst case & Original & Reordered & Original & Reordered & Original & Reordered \\
\hline Uniform mesh 1 & 20.1 & 528.0 & 22.0 & & 0.0 & & 292 & \\
\hline Uniform mesh 2 & 20.1 & 528.0 & 21.9 & & 0.0 & & 292 & \\
\hline Cardiac mesh 1 & 20.5 & 528.0 & 170.1 & 21.6 & 2.7 & 0.0 & 27 & 271 \\
\hline Cardiac mesh 20 & 20.3 & 528.0 & 250.4 & 22.4 & 3.2 & 0.0 & 22 & 260 \\
\hline Cardiac mesh 41 & 20.4 & 528.0 & 229.8 & 22.3 & 3.1 & 0.0 & 23 & 260 \\
\hline Cardiac mesh 44 & 20.4 & 528.0 & 217.9 & 22.0 & 3.0 & 0.0 & 24 & 251 \\
\hline Aneurysm mesh 3 & 20.0 & 528.0 & 33.7 & 20.6 & 0.5 & 0.0 & 63 & 281 \\
\hline Aneurysm mesh 4 & 19.9 & 528.0 & 65.2 & 21.2 & 0.7 & 0.0 & 49 & 268 \\
\hline
\end{tabular}

Aneurisk project [Aneurisk-Team 2012]. Finally, we also use two standard, uniform meshes of the unit cube. See Table 2 for an overview of the meshes.

\subsection{Benchmarking Assembly Kernels}

In this section, we report the results of benchmarking the individual assembly kernels from Section 3 on a single core. Parallel benchmarks follow in the next section.

6.2.1 Gathering Vertex Coordinates. We begin by benchmarking the first assembly kernel, which is gathering the vertex coordinates of a mesh. Table 3 shows the single-core performance for each mesh, both before and after the mesh has been reordered, as described in Section 4.1. The original mesh ordering is the one output by the mesh generator, and it has a rather poor data locality compared to the reordered version. The best- and worst case columns show best- and worst case estimates of the memory traffic, as described in Section 5.2.

The results show that reordering yields a speedup for the cellwise algorithm of about 1011 times for the cardiac meshes and about 4-5 times for the aneurysm meshes. Besides the performance, we have also measured the memory traffic by using the hardware performance events skx_unc_imc[0-5]: :UNC_M_CAS_COUNT.RD that are associated with the CPU's memory controllers. These measurements show that the memory traffic for some of the original meshes is near the worst-case estimates that were derived in Section 5.2, whereas the reordered meshes yield memory traffic volumes close to the best-case estimates. In fact, for cellwise assembly, the original cardiac meshes generate about 8-10 times more memory traffic than the reordered versions, and about 1.7-3 times more for the aneurysm meshes.

In addition, the random access patterns associated with the original meshes trigger a large number of page walks, as measured by the hardware performance event 
Table 4. Single-core Performance (in Mcell/s) for Computing Transformations to a Reference Cell and Element Matrices for the Laplacian on Intel Xeon Gold 6130

\begin{tabular}{lrrrr}
\hline & & \multicolumn{3}{l}{ Performance [Mcell/s] } \\
\cline { 3 - 5 } & flop/cell & Best case & Auto & Manual \\
\hline Transform to & 41 & $2,731.7$ & 204.5 & $1,302.8$ \\
reference cell & 60 & $1,866.7$ & 125.3 & 672.5 \\
$\mathcal{P}_{1}$ Laplacian & 116 & 965.5 & 59.8 & 208.9 \\
$\mathcal{P}_{1}$ Laplacian, variable coeff. & 394 & 284.3 & 25.2 & 167.9 \\
$\mathcal{P}_{2}$ Laplacian & & & & \\
\hline
\end{tabular}

DTLB_LOAD_MISSES.WALK_COMPLETED. These page walks occur due to missing the translation lookaside buffers, or TLBs, which are used to cache page table entries for translating virtual to physical memory addresses. Not only do page walks trigger additional memory accesses to fetch page table information from memory, but they also increase latency and reduce throughput for those memory accesses that miss the TLBs because they cannot complete before the page table information has been fetched. However, page walks are more or less eliminated by reordering the mesh, which in part explains the improved performance.

Overall, we expect the execution time of this operation to be limited by the available memory bandwidth. In practice, the achieved throughput is almost half of the $13.0 \mathrm{~GB} / \mathrm{s}$ single-core bandwidth that is measured by STREAM and reported in Table 1. It is quite plausible that hardware prefetchers are less effective when faced with irregular memory access patterns rather than the sequential memory accesses performed by STREAM. Regardless, we cannot expect to attain much better performance for the reordered meshes, since the actual volumes of memory traffic are already close to the best-case estimates and cannot be significantly improved upon.

6.2.2 Computing Element Matrices. Next, we benchmark the floating point-oriented part of the assembly procedure. We have designed this benchmark to be independent of the mesh connectivity and memory traffic concerns, by ensuring that data is only read from and written to the first-level cache. However, it does depend on the variational form that is to be assembled. In the following, we benchmark a kernel for computing the transformation to a reference mesh cell, as well as kernels for computing element matrices for the Laplacian. The single-core performance of these kernels is presented in Table 4, including default kernels that rely on compiler autovectorisation and AVX512 kernels that use manually implemented cross-element vectorisation. Here, the "Best-case" is based on the peak performance of 112 Gflop/s for a single core, given in Table 1, which assumes that FMAs are always used. In reality, the best case estimate is overly optimistic, since FMAs will only be applicable to some fraction of the operations performed. Moreover, the Laplacian kernels also involve divisions, which are much more costly than FMAs, and thus limit the overall throughput.

For the default kernels, we are relying on the compiler to automatically perform vectorisation, if possible. However, inspecting the generated assembly code reveals that it is unable to do so. Moreover, the performance ranges from 7.6 to $14.4 \mathrm{Gflop} / \mathrm{s}$. Observe that the lower number is close to the peak scalar floating point performance of a single core when fused multiply-add instructions are not used, whereas $14.4 \mathrm{Gflop} / \mathrm{s}$ is precisely the scalar floating point capacity of a single core when fused multiply-add instructions are used. Thus, the performance varies somewhat between the kernels, depending on whether or not the compiler is able to employ fused multiply-add instructions. 
We observe a significant increase in throughput for the manually implemented cross-element vectorisation that uses AVX-512 to compute eight element matrices simultaneously. More specifically, the transformation to a reference cell and the Laplacian kernels all experience a speedup of about 3.5-7 times. Also, manual vectorisation attains almost half of the peak floating point performance for the transform to reference cell, and $38 \%$ and $56 \%$ of peak floating point performance for the first- and second-order Laplacian, respectively. This should be considered a high level of utilisation, if we take into account expensive division operations and that fused multiply-add instructions may only be relevant for a portion of the overall calculations.

6.2.3 Scattering Results to a Global Matrix. The final assembly kernels involve updating a global matrix based on element matrices that are computed in the previous step. In this case, we expect the performance to be highly dependent on the mesh and finite elements that are used, and, in particular, on the numbering of both the mesh cells and the global degrees of freedom of the finite element space. The single-core performance of Algorithms 5 and 6 is shown in Table 5, for the original and reordered meshes and for first- and second-order Lagrange elements.

The first thing to note is the tremendous performance improvement that results from using a lookup table to locate non-zeros in the global matrix, rather than relying on a binary search. For first-order elements, the speedup is about 6 times when the original mesh ordering is used and about 24 times for the reordered meshes.

The performance improvement for the original mesh ordering could be partly explained by the fact that the lookup table more than halves the memory traffic compared to the binary search. Recall from our analysis of the memory traffic in Section 5.5, that the binary search results in somewhat irregular accesses to the column indices of the global matrix. Moreover, the column indices and the global matrix values must compete for space in the cache. The lookup table, on the other hand, does not access the column indices at all. Although the global matrix values are still accessed irregularly, there is more room overall for them to fit in the cache, especially in the case of a poorly ordered mesh. Thus, the lookup table leads to less memory traffic in this instance.

However, for the reordered meshes, it is in fact the lookup table that generates more memory traffic, in spite of the huge speedup that is observed. This indicates that the binary search is not at all limited by memory bandwidth in this case. More likely, the CPU is unable to ensure enough concurrent memory requests to use the memory subsystem effectively, probably due to conditional statements required in the search that incur pipeline stalls. For example, inspecting the hardware performance events skx::BR_INST_RETIRED.ALL_BRANCHES and skx: :BR_MISP_RETIRED.ALL_BRANCHES for the scattering of element matrices for "Cardiac mesh 20 ", reveals that the branch misprediction rate is $18.6 \%$ for the binary search. Unsurprisingly, the lookup table incurs virtually no branch mispredictions. Ultimately, the binary search is limited by memory latency, rather than bandwidth, a problem that is exacerbated by the fact that the binary search issues significantly more loads than the lookup table approach.

Second, for cellwise assembly with a lookup table, we observe that reordering the meshes results in a speedup of about 10-11 times for the cardiac meshes and about 5-6 times for the aneurysm meshes. This is closely correlated with a measured reduction in both read and write memory traffic. Overall, the reordering strategy works well, since the memory traffic ends up close to the best case, and there seems to be little room for improvement. Even though the memory traffic for the cardiac and aneurysm meshes ends up close to that of the uniform meshes, the performance is still noticeably better for the uniform meshes. We expect this difference is because the uniform meshes benefit more from prefetching due to more structured memory access patterns.

As expected, the row-wise algorithm is unaffected by mesh reordering. It achieves quite good performance regardless, as the cellwise algorithm is only about $5 \%-15 \%$ faster in the case of 
Table 5. Single-core Performance and Memory Traffic for Scattering Element Matrices to a Global Matrix on Intel Xeon Gold 6130

\begin{tabular}{|c|c|c|c|c|c|c|c|c|c|c|}
\hline \multirow[b]{2}{*}{ Mesh } & \multicolumn{3}{|c|}{ DRAM read [B/cell] } & \multicolumn{3}{|c|}{ DRAM write [B/cell] } & \multicolumn{2}{|c|}{ Page walks/cell } & \multicolumn{2}{|c|}{ Perf. [Mcell/s] } \\
\hline & Best case & Orig. & Reord. & Best case & Orig. & Reord. & Orig. & Reord. & Orig. & Reord. \\
\hline \multicolumn{11}{|c|}{$\mathcal{P}_{1}$, cellwise, binary search } \\
\hline Uniform mesh 1 & 47.0 & 55.6 & & 20.2 & 25.8 & & 0.0 & & 19.8 & \\
\hline Uniform mesh 2 & 46.9 & 72.1 & & 20.2 & 35.7 & & 0.0 & & 16.9 & \\
\hline Cardiac mesh 1 & 48.4 & $1,375.5$ & 59.0 & 21.1 & 516.8 & 24.8 & 8.8 & 0.0 & 1.4 & 3.6 \\
\hline Cardiac mesh 20 & 47.7 & $1,511.9$ & 68.1 & 20.7 & 524.5 & 29.8 & 9.7 & 0.0 & 1.2 & 3.6 \\
\hline Cardiac mesh 41 & 48.0 & $1,471.8$ & 66.2 & 20.8 & 526.3 & 28.9 & 9.5 & 0.0 & 1.3 & 3.6 \\
\hline Cardiac mesh 44 & 48.1 & $1,435.9$ & 62.6 & 20.9 & 516.9 & 26.7 & 9.3 & 0.0 & 1.3 & 3.6 \\
\hline Aneurysm mesh 3 & 46.9 & 611.7 & 55.4 & 20.1 & 266.5 & 23.5 & 2.9 & 0.0 & 2.5 & 3.6 \\
\hline Aneurysm mesh 4 & 46.7 & 695.0 & 61.0 & 20.1 & 289.5 & 26.1 & 3.0 & 0.0 & 2.3 & 3.7 \\
\hline \multicolumn{11}{|l|}{$\mathcal{P}_{1}$, cellwise, lookup table } \\
\hline Uniform mesh 1 & 84.2 & 90.0 & & 20.2 & 25.3 & & 0.0 & & 99.8 & \\
\hline Uniform mesh 2 & 84.2 & 104.5 & & 20.2 & 40.2 & & 0.0 & & 98.5 & \\
\hline Cardiac mesh 1 & 85.1 & 547.3 & 89.3 & 21.1 & 455.4 & 23.3 & 2.5 & 0.0 & 8.1 & 87.2 \\
\hline Cardiac mesh 20 & 84.7 & 594.4 & 95.6 & 20.7 & 499.6 & 28.4 & 2.8 & 0.0 & 7.2 & 79.6 \\
\hline Cardiac mesh 41 & 84.8 & 581.5 & 95.2 & 20.8 & 487.3 & 28.0 & 2.7 & 0.0 & 7.4 & 80.5 \\
\hline Cardiac mesh 44 & 84.9 & 574.3 & 90.7 & 20.9 & 481.1 & 24.2 & 2.7 & 0.0 & 7.5 & 84.8 \\
\hline Aneurysm mesh 3 & 84.1 & 275.6 & 88.5 & 20.1 & 198.6 & 22.4 & & 0.0 & 15.8 & 87.7 \\
\hline Aneurysm mesh 4 & 84.1 & 322.8 & 91.4 & 20.1 & 242.5 & 24.7 & 0.9 & 0.0 & 13.9 & 83.7 \\
\hline \multicolumn{11}{|c|}{$\mathcal{P}_{1}$, row-wise, lookup table } \\
\hline Uniform mesh 1 & 101.6 & 101.3 & & 20.2 & 20.4 & & 0.0 & & 83.2 & \\
\hline Uniform mesh 2 & 101.5 & 101.2 & & 20.2 & 20.3 & & 0.0 & & 82.9 & \\
\hline Cardiac mesh 1 & 102.6 & 102.3 & 102.5 & 21.1 & 21.4 & 21.7 & 0.0 & 0.0 & 76.8 & 76.8 \\
\hline Cardiac mesh 20 & 102.1 & 101.7 & 101.7 & 20.7 & 20.8 & 20.8 & 0.0 & 0.0 & 77.6 & 76.5 \\
\hline Cardiac mesh 41 & 102.3 & 101.9 & 101.9 & 20.8 & 20.9 & 21.0 & 0.0 & 0.0 & 77.8 & 76.7 \\
\hline Cardiac mesh 44 & 102.4 & 102.2 & 101.9 & 20.9 & 21.3 & 21.0 & 0.0 & 0.0 & 76.4 & 76.0 \\
\hline Aneurysm mesh 3 & 101.5 & 100.9 & 100.9 & 20.1 & 20.1 & 20.1 & 0.0 & 0.0 & 79.7 & 77.7 \\
\hline Aneurysm mesh 4 & 101.4 & 101.1 & 101.2 & 20.1 & 20.2 & 20.3 & 0.0 & 0.0 & 79.4 & 76.8 \\
\hline \multicolumn{11}{|l|}{$\mathcal{P}_{2}$, cellwise, lookup table } \\
\hline Uniform mesh 1 & 708.2 & $1,040.5$ & & 308.2 & 497.8 & & 0.1 & & 6.7 & \\
\hline Uniform mesh 2 & 707.8 & $1,035.9$ & & 307.8 & 492.7 & & 0.1 & & 6.7 & \\
\hline Cardiac mesh 1 & 713.8 & $3,300.1$ & 997.9 & 313.8 & $2,521.2$ & 469.3 & 4.9 & 0.1 & 1.2 & 6.3 \\
\hline Cardiac mesh 20 & 710.9 & $3,398.1$ & $1,059.9$ & 310.9 & $2,557.6$ & 501.6 & 1 & 0.1 & 1.1 & 6.1 \\
\hline Cardiac mesh 41 & 712.0 & $3,354.7$ & $1,050.9$ & 312.0 & $2,545.9$ & 497.0 & 5 & 0.1 & 1.2 & 6.1 \\
\hline Cardiac mesh 44 & 712.5 & $3,334.3$ & $1,034.5$ & 312.5 & $2,538.5$ & 488.8 & 5 & 0.1 & 1.3 & 6.2 \\
\hline Aneurysm mesh 3 & 708.1 & $2,707.7$ & 971.4 & 308.1 & $1,875.3$ & 446.3 & 2.6 & 0.1 & 1.6 & 6.3 \\
\hline Aneurysm mesh 4 & 707.6 & $2,701.2$ & $1,026.8$ & 307.6 & $1,869.2$ & 478.5 & 2.6 & 0.1 & 1.7 & 6.1 \\
\hline \multicolumn{11}{|c|}{$\mathcal{P}_{2}$, row-wise, lookup table } \\
\hline Uniform mesh 1 & 735.0 & 756.3 & & 308.2 & 309.1 & & 0.0 & & 13.5 & \\
\hline Uniform mesh 2 & 734.6 & 755.6 & & 307.8 & 308.4 & & 0.0 & & 13.5 & \\
\hline Cardiac mesh 1 & 741.0 & 762.5 & 761.9 & 313.8 & 315.1 & 314.6 & 0.0 & 0.0 & 12.6 & 12.6 \\
\hline Cardiac mesh 20 & 737.9 & 758.9 & 760.0 & 310.9 & 311.5 & 312.2 & 0.0 & 0.0 & 12.6 & 12.7 \\
\hline Cardiac mesh 41 & 739.2 & 759.9 & 760.2 & 312.0 & 312.6 & 312.8 & 0.0 & 0.0 & 12.6 & 12.7 \\
\hline Cardiac mesh 44 & 739.7 & 760.6 & 760.6 & 312.5 & 313.3 & 313.3 & 0.0 & 0.0 & 12.6 & 12.7 \\
\hline Aneurysm mesh 3 & 734.8 & 756.1 & 756.5 & 308.1 & 309.1 & 309.5 & 0.0 & 0.0 & 12.7 & 12.7 \\
\hline Aneurysm mesh 4 & 734.3 & 755.6 & 755.7 & 307.6 & 308.5 & 308.5 & 0.0 & 0.0 & 12.7 & 12.6 \\
\hline
\end{tabular}

first-order elements. This is consistent with the fact that the row-wise algorithm requires a small amount of additional data to be read for each mesh cell, namely the mapping from global degrees of freedom to the local degrees of freedom of each mesh cell. The fact that the row-wise algorithm results in memory traffic almost identical to the best case is strong evidence of its cache-friendly nature. 
The results for second-order elements paint a similar picture. Mesh reordering results in a speedup of about 3-5 times for cellwise assembly, whereas the performance of row-wise assembly is unaffected. However, in this case, the row-wise algorithm is almost twice as fast as the cellwise approach. The former also generates less read and write memory traffic, although the observed speedup seems larger than one would expect, if it were based only on the difference in memory traffic.

\subsection{Parallel Finite Element Assembly}

Having considered each of the assembly kernels individually, we now turn our attention to the performance of the full cellwise and row-wise assembly algorithms, which involves all of the steps in Algorithms 1 and 7, respectively.

First, as a way of validating the serial performance of the cellwise assembly, we have also compared our results to the serial performance of the open-source finite element code in FEniCS [Logg et al. 2012]. Note that FEniCS uses a cellwise assembly algorithm with the binary search strategy to locate global matrix entries after each element matrix has been computed. To make a fair comparison, we ran our benchmark for a cellwise assembly algorithm to assemble the Laplacian for first-order Lagrange elements, also using the binary search strategy. The performance of our benchmark for the reordered version of "Cardiac mesh 20" is $3.53 \mathrm{Mcell} / \mathrm{s}$, whereas the performance of FEniCS is $2.37 \mathrm{Mcell} / \mathrm{s}$ with the same mesh. The performance of the two implementations is not too different, especially considering that FEniCS is a much more general code that is designed to handle more advanced use cases than our simple benchmark.

Now, we replace the binary search with our lookup table strategy, and we consider the full assembly performance using both sockets on the Intel Xeon Gold 6130 system. Tables 6 and 7 show the serial and parallel performance of the cellwise and row-wise algorithms with the reordered meshes for the mass matrix and Laplacian, including first- and second-order elements.

Let us begin with the serial performance, as shown in the leftmost columns of Tables 6 and 7. Recall that the assembly of the mass matrix and Laplacian differ only in the calculation of the element matrices. So, the slightly lower performance for assembling the Laplacian compared to the mass matrix is because the former requires more arithmetic operations.

Second, recall that the row-wise algorithm is required to gather cell vertex coordinates and compute the transformation to a reference cell several more times than the cellwise algorithm, as described in Section 4.5. This explains why the cellwise algorithm is 3 times faster than the rowwise method in the serial case, at least for first-order elements. For second-order elements, the difference in performance is much smaller. Here, the row-wise algorithm is about $5 \%-10 \%$ faster when assembling the mass matrix, but the cellwise algorithm is about $30 \%$ faster at assembling the Laplacian. Because of the much larger element matrices associated with second-order elements, it seems clear that the scattering kernel is more dominant than it is for first-order elements. Thus, the row-wise assembly algorithm suffers less from the additional work it must do in connection with gathering vertex coordinates and transforming to a reference cell. In any case, the cellwise assembly algorithm is preferable for a sequential assembly, particularly for first-order elements.

Turning to the parallel performance, notice that the parallel, cellwise assembly experiences some slowdown at first compared to the serial algorithm. For example, in the case of first-order elements, eight threads or more are typically needed for the parallel, cellwise assembly to be faster than the serial algorithm. For second-order elements, four threads is sufficient. In any case, the cellwise algorithm scales reasonably well after that, and the performance reaches about 200-240 Mcell/s for first-order elements and 32-35 Mcell/s for second-order elements.

Similarly, we observe that row-wise assembly becomes faster than single-threaded cellwise assembly whenever four or more threads are used. The row-wise algorithm also scales nicely 
Table 6. Parallel Performance (in Mcell/s) and Main Memory Throughput (in GB/s) of Cellwise Finite Element Assembly on a Dual Socket Intel Xeon Gold 6130

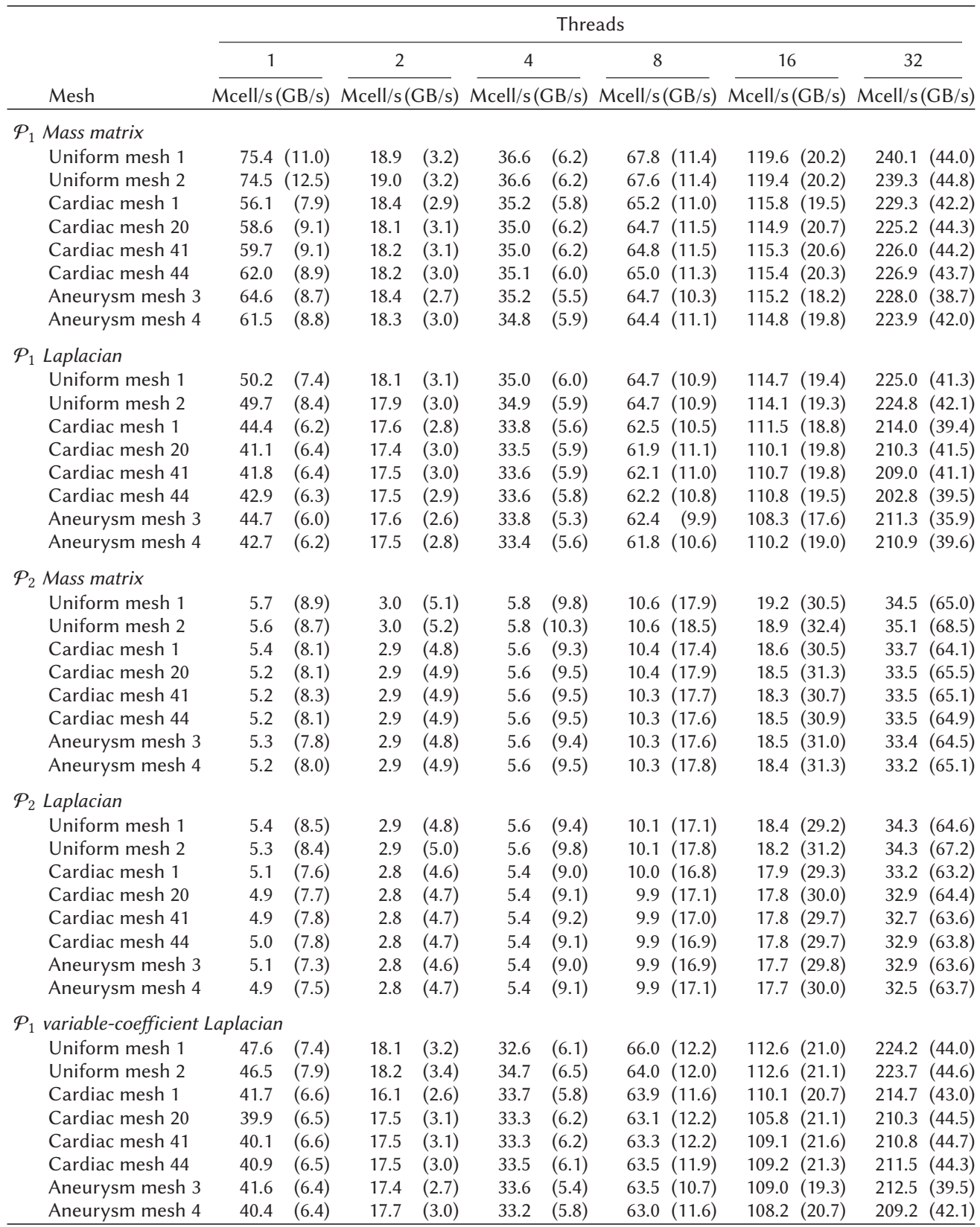

as the number of threads increases. In the end, using 32 threads to assemble the mass matrix with first-order elements, the performance reaches about $750 \mathrm{Mcell} / \mathrm{s}$ for the uniform meshes and about $500 \mathrm{Mcell} / \mathrm{s}$ for the other meshes. The performance for the Laplacian is slightly lower at about $430 \mathrm{Mcell} / \mathrm{s}$ for the uniform meshes and 310-350 Mcell/s for the other meshes. The higher 
Table 7. Parallel Performance (in Mcell/s) and Main Memory Throughput (in GB/s) of Row-wise Finite Element Assembly on a Dual Socket Intel Xeon Gold 6130

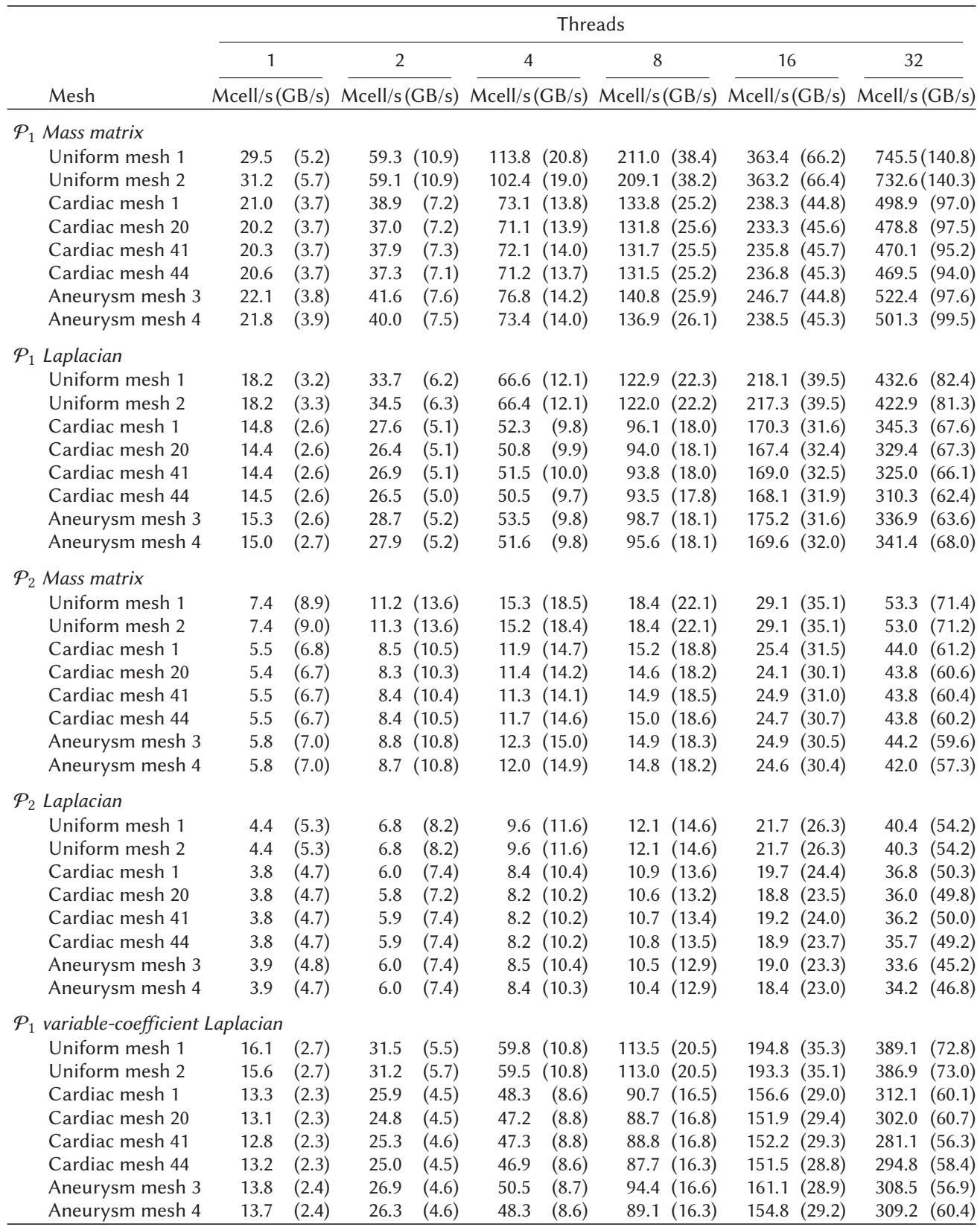

performance observed for the uniform meshes is likely because the memory access patterns are regular enough to benefit from hardware prefetchers in this case.

Overall, the row-wise algorithm outperforms the cellwise algorithm. Recall that the latter method suffers from synchronisation overhead associated with atomics. These are needed to avoid race conditions, but prevent efficient use of the memory bandwidth. The achieved main memory 
throughput for cellwise assembly with first-order elements, shown in Table 6, is only about $30 \%$ of the $147.1 \mathrm{~GB} / \mathrm{s}$ dual-socket memory bandwidth of the Intel Xeon. Compare this to the throughput for row-wise assembly, shown in Table 7, which is significantly higher, although it varies depending on the mesh and variational form. That is, for the uniform meshes, the throughput is $95 \%$ and $56 \%$ of the memory bandwidth for the mass matrix and Laplacian, respectively. For the other meshes, the throughput is about $67 \%$ for the mass matrix and about $45 \%$ for the Laplacian. The high level of bandwidth utilisation shown here combined with the results of Section 6.2, which show that the memory traffic volumes are fairly close to the theoretical minimum values, demonstrating that the performance of the row-wise assembly algorithm is close to ideal. In particular, one cannot hope to do any better in the case of assembling a mass matrix using first-order elements and a uniform mesh.

For second-order elements, the situation is slightly more complex, but the throughputs of the cellwise and row-wise approaches are roughly comparable and lie between $30 \%-50 \%$ of the memory bandwidth. There are more arithmetic operations involved for second-order elements, so it is unlikely that arithmetic operations and memory accesses will overlap perfectly. At least in the case of row-wise assembly, arithmetic operations have a larger performance impact, and so the effective throughput is a bit lower compared to first-order elements.

In summary, the parallel, row-wise assembly algorithm makes good use of the memory bandwidth, thereby achieving higher performance than the cellwise approach. We note, however, that the success of the row-wise approach depends to some extent on the first steps of the assembly algorithm-that is, gathering vertex coordinates, transforming to a reference cell and computing element matrices-being cheaper than the final step of scattering element matrices. This is evidently the case when assembling the mass matrix or the Laplacian, as we have shown here. One might expect the advantages of row-wise assembly to dwindle when assembling more complex operators. For instance, the assembly of some operators is dominated much more by arithmetic operations, whereas other operators require more input data, such as variable coefficients. A thorough investigation of row-wise assembly for more complicated operators is beyond the scope of the current article.

Finally, to provide a more complete picture, Table 8 shows the parallel performance of a rowwise assembly for the Laplacian with first-order elements on three different multi-core systems, including Intel Xeon Gold 6130, AMD Epyc 7601 and Cavium TX2 CN9980. For the latter two systems, the serial performance lags behind Intel Xeon. This is partly due to the CPU cores operating at a lower frequency than the Intel Xeon, although the Cavium TX2 also seems less able to make use of instruction-level parallelism. The row-wise assembly reaches about 340-400 Mcell/s and 310-380 Mcell/s on AMD Epyc and Cavium TX2, respectively.

A visual comparison of the performance of the three systems is shown in Figure 2 for "Cardiac mesh 20". In the whole-system dual-socket case, AMD Epyc is faster than both Intel Xeon and Cavium TX2. The performance of the latter two systems is comparable, in spite of Cavium TX2 having twice as many cores and a significantly higher memory bandwidth. Thus, the finite element assembly performance is contrary to the STREAM Triad results in Table 1, which show that Cavium TX2 has the highest single- and dual-socket memory bandwidth of the three systems. This leads us to conclude that performance on Cavium TX2 is not primarily limited by the available memory bandwidth, and perhaps there is some opportunity to further optimise instruction-level parallelism and thus improve memory bandwidth usage.

\section{RELATED WORK}

The standard cellwise assembly algorithm is described, for example, by Ern and Guermond [2004], and in the FEniCS book [Logg et al. 2012]. The tensor representation for computing element 
Table 8. Parallel Performance (in Mcell/s) of Row-wise Finite Element Assembly for the Laplacian with First-order Lagrange Elements Systems

\begin{tabular}{lrrrrrrr}
\hline & \multicolumn{7}{c}{ Threads vs. row-wise performance [Mcell/s] } \\
\cline { 2 - 8 } Mesh & 1 & 2 & 4 & 8 & 16 & 32 & 64 \\
\hline Intel Xeon Gold 6130 & & & & & & & \\
Uniform mesh 1 & 18.2 & 33.7 & 66.6 & 122.9 & 218.1 & 432.6 & \\
Uniform mesh 2 & 18.2 & 34.5 & 66.4 & 122.0 & 217.3 & 422.9 & \\
Cardiac mesh 1 & 14.8 & 27.6 & 52.3 & 96.1 & 170.3 & 345.3 & \\
Cardiac mesh 20 & 14.4 & 26.4 & 50.8 & 94.0 & 167.4 & 329.4 & \\
Cardiac mesh 41 & 14.4 & 26.9 & 51.5 & 93.8 & 169.0 & 325.0 & \\
Cardiac mesh 44 & 14.5 & 26.5 & 50.5 & 93.5 & 168.1 & 310.3 & \\
Aneurysm mesh 3 & 15.3 & 28.7 & 53.5 & 98.7 & 175.2 & 336.9 & \\
Aneurysm mesh 4 & 15.0 & 27.9 & 51.6 & 95.6 & 169.6 & 341.4 & \\
AMD Epyc 7601 & & & & & & & \\
Uniform mesh 1 & 11.9 & 23.3 & 45.2 & 86.4 & 165.4 & 292.1 & 394.9 \\
Uniform mesh 2 & 12.3 & 23.3 & 45.2 & 88.6 & 173.6 & 321.2 & 387.3 \\
Cardiac mesh 1 & 11.2 & 20.4 & 37.9 & 72.2 & 133.3 & 246.2 & 383.4 \\
Cardiac mesh 20 & 10.5 & 20.4 & 37.4 & 71.8 & 134.7 & 247.3 & 393.2 \\
Cardiac mesh 41 & 10.6 & 19.7 & 37.4 & 71.5 & 135.6 & 255.4 & 383.1 \\
Cardiac mesh 44 & 10.8 & 20.3 & 36.8 & 71.0 & 137.1 & 256.6 & 350.1 \\
Aneurysm mesh 3 & 10.7 & 21.1 & 38.9 & 75.1 & 139.0 & 253.0 & 338.2 \\
Aneurysm mesh 4 & 11.4 & 21.0 & 38.4 & 73.6 & 140.8 & 262.0 & 403.1 \\
Cavium TX2 CN9980 & & & & & & & \\
Uniform mesh 1 & 6.5 & 12.8 & 24.7 & 49.2 & 97.8 & 193.9 & 382.4 \\
Uniform mesh 2 & 6.3 & 12.3 & 24.3 & 48.3 & 96.6 & 191.4 & 381.7 \\
Cardiac mesh 1 & 6.1 & 12.1 & 23.4 & 45.4 & 87.8 & 171.9 & 340.3 \\
Cardiac mesh 20 & 6.0 & 11.6 & 22.8 & 44.4 & 86.3 & 168.3 & 320.9 \\
Cardiac mesh 41 & 6.0 & 11.9 & 23.0 & 43.8 & 87.1 & 167.7 & 320.9 \\
Cardiac mesh 44 & 6.0 & 11.8 & 22.8 & 44.4 & 86.0 & 167.3 & 312.5 \\
Aneurysm mesh 3 & 6.1 & 12.1 & 24.1 & 46.8 & 89.6 & 172.2 & 328.3 \\
Aneurysm mesh 4 & 6.1 & 11.9 & 22.7 & 44.1 & 85.8 & 167.8 & 327.7 \\
\hline & & & & & & &
\end{tabular}

matrices is described by Kirby et al. [2005] and Kirby and Logg [2006], and it was extended to more general finite elements and variational forms by Rognes et al. [2009]. Besides the tensor representation, the optimisation of kernels for computing element matrices has been studied from several different angles in connection with automated code generation for FEniCS [Logg et al. 2012] and Firedrake [Rathgeber et al. 2016]. This includes numerical integration [Ølgaard and Wells 2010], the use of computer algebra for exact integration and simplification of expressions [Alnæs and Mardal 2010; Russell and Kelly 2013], as well as low-level loop optimisations [Homolya et al. 2018; Luporini et al. 2017, 2015].

A lot of work on optimising finite element assembly has focused on reducing the number of floating point operations that are required to compute element matrices, for instance, using a technique called sum factorisation [Bolis et al. 2014; Cantwell et al. 2011; Kronbichler and Kormann 2012; Vos et al. 2010], which exploits tensor-product structure that is particularly relevant for quadrilateral and hexahedral meshes and higher-order polynomial spaces. Sometimes, a fully assembled global 


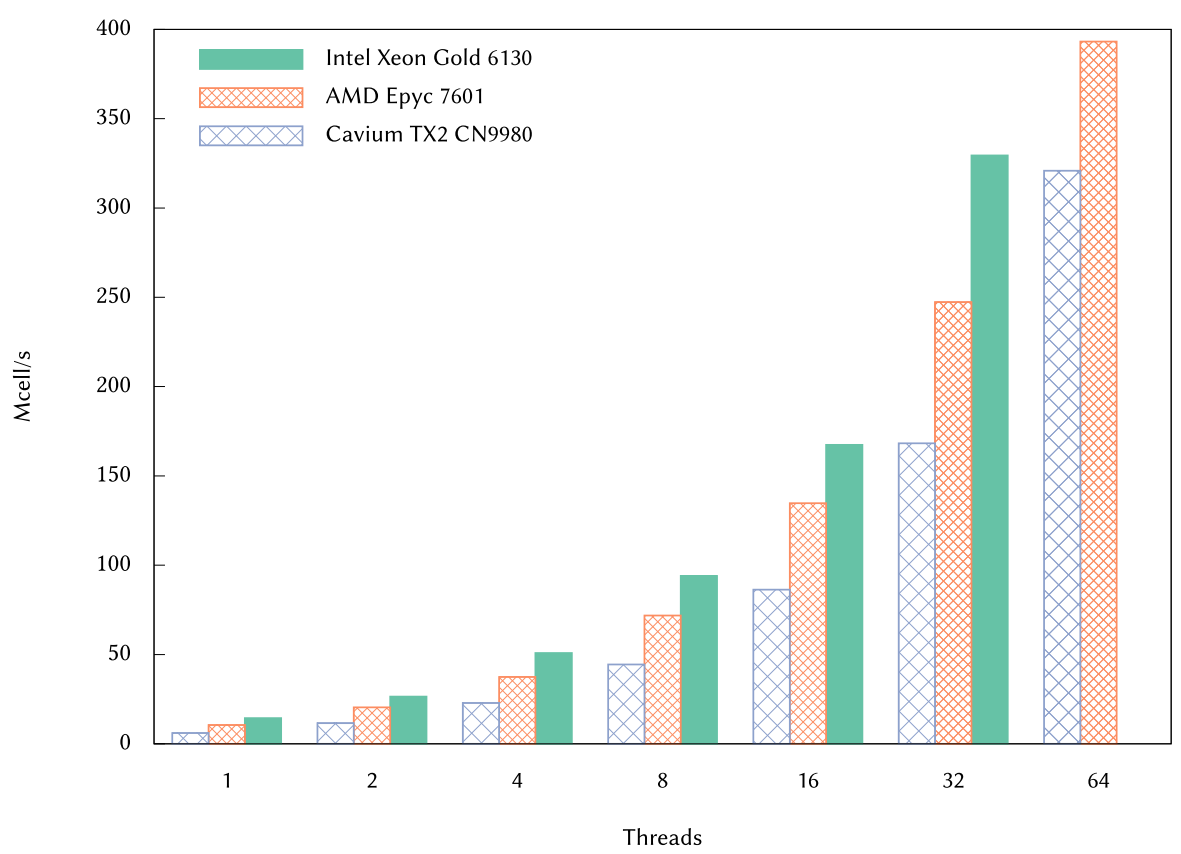

Fig. 2. Parallel performance for row-wise assembly of the Laplacian with first-order Lagrange elements for "Cardiac mesh 20".

matrix itself is not needed, but it is sufficient to evaluate the product of such a matrix with a vector. In these circumstances, it is possible to use matrix-free methods [Kronbichler and Kormann 2012] or variations, such as the local matrix approach [Cantwell et al. 2011; Markall et al. 2013; Vos et al. 2010].

Sun et al. [2020] describe the use of cross-element vectorisation on Intel multi-core CPUs using AVX-512, and demonstrate some significant performance improvements for more complicated variational forms. Other works that employ cross-element vectorisation include Fischer et al. [2020]; Kronbichler and Kormann [2012]; Moxey et al. [2020], and also a similar idea is used by Knepley and Terrel [2013] in their implementation of a GPU-based element matrix computation. Brown [2010] describes the use of manual vectorisation to speed up the calculation of individual element matrices within the context of higher-order, matrix-free implementations for hexahedral elements. Another example of combining matrix-free methods and vectorisation is given by Kronbichler and Kormann [2019]. They also describe different choices for the geometry-related parts of element matrix calculation, such as precomputing the Jacobian, its inverse and determinant, and reusing these quantities for repeated assembly of element matrices.

More recently, a lot of work has focused on implementing finite element assembly algorithms for GPUs. Cecka et al. [2011] explored several strategies for global assembly, including both cellwise and row-wise algorithms. Markall et al. [2013] compare parallel, global matrix assembly, where race conditions are avoided by using mesh colouring or atomics, to what they call a local matrix approach. The latter is like a matrix-free method except that the element matrices are computed only once, and they are stored in memory and reused each time a matrix-vector multiplication is computed. Fu et al. [2014] implement a cellwise assembly algorithm that uses a binary search when scattering element matrices to a global matrix. Their method also relies on partitioning the mesh to ensure that data fits in a GPU's fast shared memory. Reguly and Giles [2015] perform a detailed 
comparison of both the assembly and solution of linear systems, where they compare cellwise, global matrix assembly for CSR and ELLPACK sparse matrix formats to the local matrix- and matrix-free methods. In addition to various experiments that demonstrate the tradeoffs between different methods, they also describe the memory traffic required by each method.

\section{CONCLUSION}

Finite element assembly algorithms with linear or quadratic finite elements on unstructured meshes are characterised by low arithmetic intensity and irregular memory access patterns. As a result, moving data to and from memory can greatly impact the performance. We have performed a detailed breakdown of the standard cellwise finite element assembly algorithm, focusing on a quantified understanding of the memory traffic involved. Estimated lower and upper bounds of the memory traffic have been derived and used to demonstrate the effectiveness of some optimisations related to, specifically, mesh reordering and use of a lookup table in the scattering kernel. In addition, we have shown that a row-wise assembly algorithm, which has previously been used for finite element assembly on GPUs, is also an efficient strategy for parallel assembly with shared memory on multi-core CPUs.

\section{ACKNOWLEDGMENTS}

We would like to thank Hermenegild Arevalo and Alban Souche for their help in obtaining unstructured computational meshes that were used in this study.

\section{REFERENCES}

Martin Sandve Alnæs and Kent-André Mardal. 2010. On the efficiency of symbolic computations combined with code generation for finite element methods. ACM Transactions on Mathematical Software 37, 1 (2010), 26 pages. DOI:https: //doi.org/10.1145/1644001.1644007

Aneurisk-Team. 2012. AneuriskWeb Project Website. (June 2012). Retrieved June 9, 2020 from http://ecm2.mathcs.emory. edu/aneuriskweb.

A. Bolis, C. D. Cantwell, R. M. Kirby, and S. J. Sherwin. 2014. From $h$ to $p$ efficiently: Optimal implementation strategies for explicit time-dependent problems using the spectral/hp element method. International fournal for Numerical Methods in Fluids 75, 8 (2014), 591-607. DOI:https://doi.org/10.1002/fld.3909

Jed Brown. 2010. Efficient nonlinear solvers for nodal high-order finite elements in 3D. Journal of Scientific Computing 45, 1-3 (2010), 48-63. DOI:https://doi.org/10.1007/s10915-010-9396-8

C. D. Cantwell, S. J. Sherwin, R. M. Kirby, and P. H. J. Kelly. 2011. From h to p efficiently: Strategy selection for operator evaluation on hexahedral and tetrahedral elements. Computers \& Fluids 43, 1 (2011), 23-28. DOI:https://doi.org/10.1016/ j.compfluid.2010.08.012

G. F. Carey, E. Barragy, R. McLay, and M. Sharma. 1988. Element-by-element vector and parallel computations. Communications in Applied Numerical Methods 4, 3 (1988), 299-307. DOI:https://doi.org/10.1002/cnm.1630040303

Cris Cecka, Adrian J. Lew, and E. Darve. 2011. Assembly of finite element methods on graphics processors. International fournal for Numerical Methods in Engineering 85, 5 (2011), 640-669. DOI:https://doi.org/10.1002/nme.2989

Philippe G. Ciarlet. 2002. The Finite Element Method for Elliptic Problems. Society for Industrial and Applied Mathematics, Philadelphia. DOI:https://doi.org/10.1137/1.9780898719208

E. Cuthill and J. McKee. 1969. Reducing the bandwidth of sparse symmetric matrices. In Proceedings of the 1969 24th National Conference. Association for Computing Machinery, New York, NY, 157-172. DOI:https://doi.org/10.1145/800195.805928

Alexandre Ern and Jean-Luc Guermond. 2004. Theory and Practice of Finite Elements. Springer, New York. DOI:https://doi. org/10.1007/978-1-4757-4355-5

Paul Fischer, Misun Min, Thilina Rathnayake, Som Dutta, Tzanio Kolev, Veselin Dobrev, Jean-Sylvain Camier, Martin Kronbichler, Tim Warburton, Kasia Wirydowicz, and Jed Brown. 2020. Scalability of high-performance PDE solvers. The International fournal of High Performance Computing Applications 34, 5 (2020), 562-586. DOI:https://doi.org/10.1177/ 1094342020915762

Matteo Frigo, Charles E. Leiserson, Harald Prokop, and Sridhar Ramachandran. 2012. Cache-oblivious algorithms. ACM Transactions on Algorithms 8, 1 (2012), 22 pages. DOI:https://doi.org/10.1145/2071379.2071383

Zhisong Fu, T. James Lewis, Robert M. Kirby, and Ross T. Whitaker. 2014. Architecting the finite element method pipeline for the GPU. Journal of Computational and Applied Mathematics 257 (2014), 195-211. DOI:https://doi.org/10.1016/j.cam. 2013.09.001 
Alan George. 1973. Nested dissection of a regular finite element mesh. SIAM Journal on Numerical Analysis 10, 2 (1973), 345-363. DOI:https://doi.org/10.1137/0710032

Alan George and David R. Mcintyre. 1978. On the application of the minimum degree algorithm to finite element systems. SIAM Journal on Numerical Analysis 15, 1 (1978), 90-112. DOI : https://doi.org/10.1007/BFb0064460

Xu Guo. 2019. Best Practice Guide - AMD EPYC. (Feb. 2019). Retrieved June 9, 2020 from https://prace-ri.eu/wp-content/ uploads/Best-Practice-Guide_AMD.pdf.

Miklós Homolya, Lawrence Mitchell, Fabio Luporini, and David A. Ham. 2018. TSFC: A structure-preserving form compiler. SIAM Journal on Scientific Computing 40, 3 (2018), 401-428. DOI:https://doi.org/10.1137/17M1130642

Intel Corporation. 2018. Intel ${ }^{\circledR} 64$ and IA-32 Architectures Optimization Reference Manual. (April 2018). Retrieved June 9, 2020 from https://software.intel.com/content/dam/develop/public/us/en/documents/64-ia-32-architecturesoptimization-manual.pdf.

Reza Jabbari, Thomas Engstrøm, Charlotte Glinge, Bjarke Risgaard, Javad Jabbari, Bo Gregers Winkel, Christian Juhl Terkelsen, Hans-Henrik Tilsted, Lisette Okkels Jensen, Mikkel Hougaard, Stephanie E Chiuve, Frants Pedersen, Jesper Hastrup Svendsen, Stig Haunsø, Christine M Albert, and Jacob Tfelt-Hansen. 2015. Incidence and risk factors of ventricular fibrillation before primary angioplasty in patients with first ST-elevation myocardial infarction: A nationwide study in Denmark. Journal of the American Heart Association 4, 1 (2015), e001399. DOI:https://doi.org/10.1161/ JAHA.114.001399

Robert C. Kirby, Matthew Knepley, Anders Logg, and L. Ridgway Scott. 2005. Optimizing the evaluation of finite element matrices. SIAM fournal on Scientific Computing 27, 3 (2005), 741-758. DOI:https://doi.org/10.1137/040607824

Robert C. Kirby and Anders Logg. 2006. A compiler for variational forms. ACM Transactions on Mathematical Software 32 3 (2006), 417-444. DOI:https://doi.org/10.1145/1163641.1163644

Matthew G. Knepley and Andy R. Terrel. 2013. Finite element integration on GPUs. ACM Transactions on Mathematical Software 39, 2 (2013), 13. DOI: https://doi.org/10.1145/2427023.2427027

Martin Kronbichler and Katharina Kormann. 2012. A generic interface for parallel cell-based finite element operator application. Computers and Fluids 63 (2012), 135-147. DOI:https://doi.org/10.1016/j.compfluid.2012.04.012

Martin Kronbichler and Katharina Kormann. 2019. Fast matrix-free evaluation of discontinuous galerkin finite element operators. ACM Transactions on Mathematical Software 45, 3 (2019), 1-40. DOI:https://doi.org/10.1145/3325864

Anders Logg, Kent-Andre Mardal, and Garth N. Wells. 2012. Automated Solution of Differential Equations by the Finite Element Method. Springer, Berlin. DOI:https://doi.org/10.1007/978-3-642-23099-8

Fabio Luporini, David A. Ham, and Paul H. J. Kelly. 2017. An algorithm for the optimization of finite element integration loops. ACM Transactions on Mathematical Software 44, 1 (2017), 26 pages. DOI:https://doi.org/10.1145/3054944

Fabio Luporini, Ana Lucia Varbanescu, Florian Rathgeber, Gheorghe-Teodor Bercea, J. Ramanujam, David A. Ham, and Paul H. J. Kelly. 2015. Cross-loop optimization of arithmetic intensity for finite element local assembly. ACM Transactions on Architecture and Code Optimization 11, 4 (2015), 25 pages. DOI: https://doi.org/10.1145/2687415

Maciej Marciniak, Hermenegild Arevalo, Jacob Tfelt-Hansen, Thomas Jespersen, Reza Jabbari, Charlotte Glinge, Kiril A Ahtarovski, Niels Vejlstrup, Thomas Engstrom, Mary M. Maleckar, and Kristin McLeod. 2017. From CMR image to patient-specific simulation and population-based analysis: Tutorial for an openly available image-processing pipeline. In Proceedings of the STACOM 2016: Statistical Atlases and Computational Models of the Heart. Imaging and Modelling Challenges, Tommaso Mansi, Kristin McLeod, Mihaela Pop, Kawal Rhode, Maxime Sermesant, and Alistair Young (Eds.), Springer International Publishing, 106-117. DOI:https://doi.org/10.1007/978-3-319-52718-5_12

G. R. Markall, A. Slemmer, D. A. Ham, P. H. J. Kelly, C. D. Cantwell, and S. J. Sherwin. 2013. Finite element assembly strategies on multi-core and many-core architectures. International fournal for Numerical Methods in Fluids 71, 1 (2013), 80-97. DOI:https://doi.org/10.1002/fld.3648

John D. McCalpin. 2013. STREAM: Sustainable Memory Bandwidth in High Performance Computers. (Jan. 2013). Retrieved June 9, 2020 from https:/www.cs.virginia.edu/stream/.

David Moxey, Roman Amici, and Mike Kirby. 2020. Efficient matrix-free high-order finite element evaluation for simplicial elements. SIAM fournal on Scientific Computing 42, 3 (2020), C97-C123. DOI:https://doi.org/10.1137/19M1246523

Kristian B. Ølgaard and Garth N. Wells. 2010. Optimizations for quadrature representations of finite element tensors through automated code generation. ACM Transactions on Mathematical Software 37, 1 (2010), 23 pages. DOI: https: //doi.org/10.1145/1644001.1644009

Florian Rathgeber, David A. Ham, Lawrence Mitchell, Michael Lange, Fabio Luporini, Andrew T. T. Mcrae, Gheorghe-Teodor Bercea, Graham R. Markall, and Paul H. J. Kelly. 2016. Firedrake: Automating the finite element method by composing abstractions. ACM Transactions on Mathematical Software 43, 3 (2016), 27 pages. DOI: https://doi.org/10.1145/2998441

I. Z. Reguly and M. B. Giles. 2015. Finite element algorithms and data structures on graphical processing units. International fournal of Parallel Programming 43, 2 (2015), 203-239. DOI: https://doi.org/10.1007/s10766-013-0301-6

Marie E. Rognes, Robert C. Kirby, and Anders Logg. 2009. Efficient assembly of $H$ (div) and $H$ (curl) conforming finite elements. SIAM fournal on Scientific Computing 31, 6 (2009), 4130-4151. DOI: https://doi.org/10.1137/08073901X 
Francis P. Russell and Paul H. J. Kelly. 2013. Optimized code generation for finite element local assembly using symbolic manipulation. ACM Trans. Math. Software 39, 4 (2013), 29 pages. DOI: https://doi.org/10.1145/2491491.2491496

David Schor. 2018. A Look at Cavium's New High-Performance ARM Microprocessors and the Isambard Supercomputer. (June 2018). Retrieved May 22, 2020 from https://fuse.wikichip.org/news/1316/a-look-at-caviums-new-highperformance-arm-microprocessors-and-the-isambard-supercomputer/.

Tianjiao Sun, Lawrence Mitchell, Kaushik Kulkarni, Andreas Klöckner, David A. Ham, and Paul H. J. Kelly. 2020. A study of vectorization for matrix-free finite element methods. The International fournal of High Performance Computing Applications 34, 6 (2020), 629-644. DOI : https://doi.org/10.1177/1094342020945005

Marc Tchiboukdjian, Vincent Danjean, and Bruno Raffin. 2008. A fast cache-oblivious mesh layout with theoretical guarantees. In Proceedings of the International Workshop on Super Visualization. Kos, Greece. Retrieved from https: //hal.inria.fr/inria-00436053.

M. Tchiboukdjian, V. Danjean, and B. Raffin. 2010. Binary mesh partitioning for cache-efficient visualization. IEEE Transactions on Visualization and Computer Graphics 16, 5 (2010), 815-828. DOI: https://doi.org/10.1109/TVCG.2010.19

Peter E. J. Vos, Spencer J. Sherwin, and Robert M. Kirby. 2010. From $h$ to $p$ efficiently: Implementing finite and spectral $/ h p$ element methods to achieve optimal performance for low- and high-order discretisations. fournal of Computational Physics 229, 13 (2010), 5161-5181. DOI: https://doi.org/10.1016/j.jcp.2010.03.031

S.-E. Yoon and P. Lindstrom. 2006. Mesh layouts for block-based caches. IEEE Transactions on Visualization and Computer Graphics 12, 5 (2006), 1213-1220. DOI: https://doi.org/10.1109/TVCG.2006.162

Received June 2020; revised June 2021; accepted December 2021 